\title{
Synthesis of Fluorescently Labeled Mono- and Doubly Prenylated Rab7 GTPase
}

\author{
Thomas Durek ${ }^{[a]}$, Kirill Alexandrov ${ }^{[a]}$, Roger S. Goody ${ }^{[a],[b]}$, Alexandra Hildebrand ${ }^{[c]}$, Ines \\ Heinemann $n^{[b],[d]}$, and Herbert Waldmann $n^{[b],[d] *}$
}

\section{SUPPORTING INFORMATION PARAGRAPH}

General Methods. Proton and carbon NMR spectra were recorded on Varian Mercury 400, Bruker DRX 500 and Varian Inova 600 spectrometers. NMR spectra were calibrated either to an internal standard of $\mathrm{Me}_{4} \mathrm{Si}$ or to the solvent signals of $\mathrm{CDCl}_{3}(7.26 \mathrm{ppm}$ and $77.00 \mathrm{ppm})$ and $\mathrm{MeOH}-\mathrm{d}_{4}$ (49.05 ppm), respectively. Coupling constants are given in $\mathrm{Hz}$. FAB-MS were recorded on a Jeol SX 102A, MALDI-MS were recorded on a Voyager-DE ${ }^{\mathrm{TM}}$ Pro BioSpectrometer from Applied Biosystems. Applied matrices are given for each particular compound. ESI-MS were measured out using a Agilent 1100 series binary pump together with a Macherey\&Nagel analytical Nucleosil C4 column and a Finnigan Thermoquest LCQ. If not otherwise stated, the following gradient programm was used: flow: $1 \mathrm{~mL} / \mathrm{min}$, solvent $\mathrm{A}: 0.1 \%$ $\mathrm{HCO}_{2} \mathrm{H}$ in $\mathrm{H}_{2} \mathrm{O}$, solvent $\mathrm{B}: 0.1 \% \mathrm{HCO}_{2} \mathrm{H}$ in $\mathrm{MeCN}, \mathrm{A} / \mathrm{B}: 50 / 50$ (0 min), 0/100 (30 min), 0/100 (40 $\mathrm{min}$ ). Melting points are reported uncorrected. Flash chromatography was carried out on columns packaged with Merck silica gel (40-64 $\mu \mathrm{m})$, and size exclusion chromatography on columns packaged with Sephadex ${ }^{\circledR}$ LH-20 (Pharmacia). TLC was carried out on Kieselgel $60 \mathrm{~F}_{254}$ aluminum sheets (Merck). All reagents were obtained from Acros, Advanced Chemtech, Aldrich, AppliChem, Avocado, Biosolve, Chimica, Fluka, Novabiochem, Senn Chemicals or Sigma. All solvents were dried and distilled using standard procedures. ${ }^{1}$ All peptide couplings were performed utilizing standard synthetic methods.

$N^{\alpha}$-Benzyloxycarbonyl- $N$-9-fluorenylmethyloxycarbonyl-L-lysine benzyl ester (Z-Lys(Fmoc)-OBzl) (2). To a solution of Z-Lys(Fmoc)-OH 1 (1.000 g, $1.99 \mathrm{mmol})$ in methanol $(20 \mathrm{~mL})$, stirring vigorously at $0^{\circ} \mathrm{C}$ under an argon atmosphere, was added a solution of $\mathrm{Cs}_{2} \mathrm{CO}_{3}(0.318 \mathrm{~g}, 0.98 \mathrm{mmol})$ in methanol $(15 \mathrm{~mL})$. The reaction mixture was warmed to room temperature and left to stir for an additional $10 \mathrm{~min}$. The solvent was removed under reduced pressure and the cesium salt was dried at high vacuum. At $0^{\circ} \mathrm{C}$, to a suspension of the cesium salt in DMF $(10 \mathrm{~mL})$ was added benzyl bromide $(4.7 \mathrm{~mL}, 39.80 \mathrm{mmol})$ and the mixture was warmed to room temperature and left to stir for $2 \mathrm{~d}$. The solvent was removed under reduced pressure and ethyl acetate/0.5 $\mathrm{N} \mathrm{NaHCO}_{3} 1: 1(100 \mathrm{~mL})$ was added. The layers were separated, and the aqueous layer was extracted with ethyl acetate $(3 \times 50 \mathrm{~mL})$. The combined organic layers were washed with brine $(50 \mathrm{~mL})$, dried over $\mathrm{Na}_{2} \mathrm{SO}_{4}$ and filtered. The solvent was removed under reduced pressure, and the residue was purified by flash chromatography on silica gel using dichloromethane/methanol $100: 1$ as eluent to yield $0.942 \mathrm{~g}(80 \%)$ of the desired product 2 as a white solid. $R_{f}=0.43$ (cyclohexane/ethyl acetate $1: 1$ ). $\mathrm{mp}=157^{\circ} \mathrm{C}$. $[\alpha]^{20}{ }_{D}=-3.9\left(c=1.0, \mathrm{CHCl}_{3}\right) .{ }^{1} \mathrm{H}$ NMR $\left(400 \mathrm{MHz}, \mathrm{CDCl}_{3}\right): \delta 7.76(\mathrm{~d}, J=8.0 \mathrm{~Hz}, 2 \mathrm{H}, \mathrm{CH} \mathrm{Fmoc})$, 7.58 (d, $J=7.2 \mathrm{~Hz}, 2 \mathrm{H}, \mathrm{CH}$ Fmoc), 7.40 (dd, $J=7.6,7.2 \mathrm{~Hz}, 2 \mathrm{H}, \mathrm{CH}$ Fmoc), 7.29-7.34 (m, $12 \mathrm{H}$, arom. $\mathrm{CH}$ ), $5.42(\mathrm{~d}, J=7.2 \mathrm{~Hz}, 1 \mathrm{H}, \mathrm{NH}), 5.07-5.22\left(\mathrm{~m}, 4 \mathrm{H}, 2{ }^{*} \mathrm{OCH}_{2}\right), 4.77(\mathrm{br}, 1 \mathrm{H}, \alpha-$ $\mathrm{CH})$, 4.36-4.45 (m, $\left.2 \mathrm{H}, \mathrm{OCH}_{2} \mathrm{Fmoc}\right), 4.20\left(\mathrm{t}, J=6.8 \mathrm{~Hz}, 1 \mathrm{H}, \mathrm{CHCH}_{2} \mathrm{O}\right), 3.04-3.16(\mathrm{~m}, 2 \mathrm{H}, \varepsilon-$ $\left.\mathrm{CH}_{2}\right), 1.78-1.92\left(\mathrm{~m}, 1 \mathrm{H}, \beta-\mathrm{CH}_{2 \mathrm{a}}\right), 1.59-1.74\left(\mathrm{~m}, 1 \mathrm{H}, \beta-\mathrm{CH}_{2 \mathrm{~b}}\right), 1.19-1.53\left(\mathrm{~m}, 4 \mathrm{H}, \delta-, \gamma-\mathrm{CH}_{2}\right) .{ }^{13} \mathrm{C}$ NMR $\left(100.7 \mathrm{MHz}, \mathrm{CDCl}_{3}\right): \delta 172.16,156.40,155.92,143.92,141.26,136.14,135.20,128.58$, $128.47,128.31,128.14,128.05,127.60,126.97,124.95,119.90,67.11,66.97,66.41,53.67$, $47.25,40.46,32.13,29.27,22.14$. HRMS (FAB; 3-NBA) $\mathrm{m} / \mathrm{z}$ : calcd for $(\mathrm{M}+\mathrm{Na})^{+}$ $\mathrm{C}_{36} \mathrm{H}_{36} \mathrm{~N}_{2} \mathrm{NaO}_{6} 615.2471$, found 615.2449. 
$N^{(\alpha)}$-Benzyloxycarbonyl-L-lysine benzyl ester (Z-Lys-OBzl) (3). At room temperature, ZLys(Fmoc)-OBzl $2(0.880 \mathrm{~g}, 1.48 \mathrm{mmol})$ was dissolved in dry dichloromethane $(25 \mathrm{~mL})$ under an argon atmosphere, and diethylamine was added dropwise $(10 \mathrm{~mL})$. The mixture was left to stir for $9 \mathrm{~h}$ and the solvent was removed by coevaporation with toluene $(2 \times 50 \mathrm{~mL})$. The residue was dried under high vacuum and purified by flash chromatography on silica gel using dichloromethane/methanol $50: 1$ followed by $5: 1$ as eluent to yield $0.491 \mathrm{~g}(90 \%)$ of the desired product 3 as a pale reddish oil. $R_{f}=0.1$ (dichloromethane/methanol 10:1). $[\alpha]^{20}{ }_{D}=-7.2(c=$ 1.5, $\left.\mathrm{CHCl}_{3}\right) .{ }^{1} \mathrm{H}$ NMR $\left(400 \mathrm{MHz}, \mathrm{CD}_{3} \mathrm{OD}\right): \delta 7.26-7.36(\mathrm{~m}, 10 \mathrm{H}$, arom. $\mathrm{CH}), 5.04-5.20(\mathrm{~m}, 4 \mathrm{H}$, $\left.2{ }^{*} \mathrm{OCH}_{2}\right), 4.22(\mathrm{dd}, J=9.4,5.0 \mathrm{~Hz}, 1 \mathrm{H}, \alpha-\mathrm{CH}), 2.85\left(\mathrm{t}, J=7.6 \mathrm{~Hz}, 2 \mathrm{H}, \varepsilon-\mathrm{CH}_{2}\right), 1.81-1.90(\mathrm{~m}$, $\left.1 \mathrm{H}, \beta-\mathrm{CH}_{2 \mathrm{a}}\right), 1.58-1.76\left(\mathrm{~m}, 3 \mathrm{H}, \beta-\mathrm{CH}_{2 \mathrm{~b}}, \delta-\mathrm{CH}_{2}\right), 1.36-1.50\left(\mathrm{~m}, 2 \mathrm{H}, \gamma-\mathrm{CH}_{2}\right) .{ }^{13} \mathrm{C}$ NMR $(100.7$ $\left.\mathrm{MHz}, \mathrm{CD}_{3} \mathrm{OD}\right): \delta 173.72,158.76,138.16,137.28,129.61,129.51,129.38,129.10,129.07$, 128.83, 67.94, 67.71, 55.32, 40.53, 31.98, 28.06, 23.84. HRMS (FAB; 3-NBA) $\mathrm{m} / \mathrm{z}$ : calcd for $(\mathrm{M}+\mathrm{H})^{+} \mathrm{C}_{21} \mathrm{H}_{27} \mathrm{~N}_{2} \mathrm{O}_{4}$ 371.1971, found 371.1945.

$\boldsymbol{N}^{(\alpha)}$-Benzyloxycarbonyl- $\boldsymbol{N}^{(\varepsilon)}$-5-dimethylaminonaphthalene-1-sulfonyl-L-lysine benzyl ester (Z-Lys(dans)-OBzl) (4). At $0^{\circ} \mathrm{C}$, to a solution of Z-Lys-OBzl $3(430 \mathrm{mg}, 1.16 \mathrm{mmol})$ in dry dichloromethane $(50 \mathrm{~mL})$ under an argon atmosphere were added dry triethylamine $(0.32$ $\mathrm{mL}, 2.32 \mathrm{mmol}$ ) and a solution of 5-dimethylaminonaphthalene-1-sulfonyl chloride (313 $\mathrm{mg}$, $1.16 \mathrm{mmol})$ in dry dichloromethane $(10 \mathrm{~mL})$. Under exclusion of light, the mixture was left to stir for $30 \mathrm{~min}$ at $0^{\circ} \mathrm{C}$, and then for $6 \mathrm{~h}$ at room temperature. The solvent was then removed by coevaporation with toluene $(2 \times 40 \mathrm{~mL})$. The residue was dried in high vacuum and purified by flash chromatography on silica gel using cyclohexane/ethyl acetate $5: 1$, followed by $4: 1$ as eluent to yield $0.501 \mathrm{~g}(72 \%)$ of the desired product 4 as a yellowish-green oil. $R_{f}=0.4$ (cyclohexane/ethyl acetate 1:1). $[\alpha]^{20}{ }_{D}=+1.8\left(c=1.0, \mathrm{CHCl}_{3}\right) .{ }^{1} \mathrm{H} \mathrm{NMR}\left(400 \mathrm{MHz}, \mathrm{CDCl}_{3}\right): \delta$ $8.53(\mathrm{~d}, J=8.4 \mathrm{~Hz}, 1 \mathrm{H}, \mathrm{CH}$ dansyl), $8.30(\mathrm{~d}, J=8.8 \mathrm{~Hz}, 1 \mathrm{H}, \mathrm{CH}$ dansyl), $8.22(\mathrm{~d}, J=7.2 \mathrm{~Hz}$, $1 \mathrm{H}, \mathrm{CH}$ dansyl), $7.53(\mathrm{dd}, J=8.2 \mathrm{~Hz}, 1 \mathrm{H}, \mathrm{CH}$ dansyl), $7.50(\mathrm{dd}, J=7.5 \mathrm{~Hz}, 1 \mathrm{H}, \mathrm{CH}$ dansyl), 7.25-7.38 (m, $10 \mathrm{H}$, arom. $\mathrm{CH}), 7.16(\mathrm{~d}, J=7.6 \mathrm{~Hz}, 1 \mathrm{H}, \mathrm{CH}$ dansyl), $5.33(\mathrm{~d}, J=8.4 \mathrm{~Hz}, 1 \mathrm{H}$, $\left.{ }^{(\alpha)} \mathrm{NH}\right), 5.07-5.17\left(\mathrm{~m}, 4 \mathrm{H}, 2\right.$ * $\left.\mathrm{OCH}_{2}\right), 5.03\left(\mathrm{t}, J=5.6 \mathrm{~Hz}, 1 \mathrm{H},{ }^{(\varepsilon)} \mathrm{NH}\right), 4.28-4.33(\mathrm{~m}, 1 \mathrm{H}, \alpha-\mathrm{CH})$, $2.87\left(\mathrm{~s}, 6 \mathrm{H}, \mathrm{N}\left(\mathrm{CH}_{3}\right)_{2}\right), 2.80\left(\mathrm{dt}, J=6.4 \mathrm{~Hz}, 2 \mathrm{H}, \varepsilon-\mathrm{CH}_{2}\right), 1.60-1.73\left(\mathrm{~m}, 1 \mathrm{H}, \beta-\mathrm{CH}_{2 \mathrm{a}}\right), 1.42-1.55$ $\left(\mathrm{m}, 1 \mathrm{H}, \beta-\mathrm{CH}_{2 \mathrm{~b}}\right), 1.07-1.42\left(\mathrm{~m}, 4 \mathrm{H}, \delta-, \gamma-\mathrm{CH}_{2}\right) .{ }^{13} \mathrm{C} \mathrm{NMR}\left(100.7 \mathrm{MHz}, \mathrm{CDCl}_{3}\right): \delta 172.01,155.90$, $151.86,136.10,135.15,134.67,130.27,129.76,129.50,128.52,128.42,128.41,128.27$, 128.25, 128.09, 128.03, 123.10, 118.68, 115.13, 67.04, 66.96, 53.45, 45.31, 42.72, 31.91, 28.73, 21.89. HRMS (FAB; 3-NBA) $\mathrm{m} / \mathrm{z}$ : calcd for $(\mathrm{M}+\mathrm{H})^{+} \mathrm{C}_{33} \mathrm{H}_{38} \mathrm{~N}_{3} \mathrm{O}_{6} \mathrm{~S} 604.2481$, found 604.2465 .

$N^{(\varepsilon)}$-5-Dimethylaminonaphthalene-1-sulfonyl-L-lysine $\quad(H-L y s(d a n s)-O H) \quad(5)$. To a solution of the proteced lysine $4(50 \mathrm{mg}, 0.08 \mathrm{mmol})$ in methanol $(5 \mathrm{~mL})$ was added a catalytic amount of palladium (10\%) on charcoal. The solution was carefully degassed, and left to stir for $6 \mathrm{~h}$ under an atmosphere of hydrogen. The reaction suspension was filtered over Celite ${ }^{\circledR}$ and the solvent was removed under reduced pressure to yield $31 \mathrm{mg}(100 \%)$ of the desired product 5 as a yellowish-green solid. $R_{f}=0.33$ (dichloromethane/methanol $2: 1$ ). $\mathrm{mp}=202^{\circ} \mathrm{C}^{2}$ ${ }^{1} \mathrm{H}$ NMR $\left(400 \mathrm{MHz}, \mathrm{CDCl}_{3} / \mathrm{CD}_{3} \mathrm{OD} 10: 1\right): \delta 8.55(\mathrm{~d}, J=8.4 \mathrm{~Hz}, 1 \mathrm{H}, \mathrm{CH}$ dansyl), $8.34(\mathrm{~d}, J=8.8$ $\mathrm{Hz}, 1 \mathrm{H}, \mathrm{CH}$ dansyl), 8.17 (dd, $J=7.4 \mathrm{~Hz}, 1.0,1 \mathrm{H}, \mathrm{CH}$ dansyl), $7.58(\mathrm{dd}, J=8.8,6.4 \mathrm{~Hz}, 1 \mathrm{H}$, $\mathrm{CH}$ dansyl), 7.56 (dd, $J=8.4,6.4 \mathrm{~Hz}, 1 \mathrm{H}, \mathrm{CH}$ dansyl), $7.27(\mathrm{~d}, J=6.8 \mathrm{~Hz}, 1 \mathrm{H}, \mathrm{CH}$ dansyl), $3.43(\mathrm{dd}, J=7.2,5.2 \mathrm{~Hz}, 1 \mathrm{H}, \alpha-\mathrm{CH}), 2.87\left(\mathrm{~s}, 6 \mathrm{H}, \mathrm{N}\left(\mathrm{CH}_{3}\right)_{2}\right), 2.83\left(\mathrm{t}, J=6.8 \mathrm{~Hz}, 2 \mathrm{H}, \varepsilon-\mathrm{CH}_{2}\right)$, 1.62-1.81 (m, $\left.2 \mathrm{H}, \beta-\mathrm{CH}_{2}\right), 1.31-1.47\left(\mathrm{~m}, 4 \mathrm{H}, \delta-, \gamma-\mathrm{CH}_{2}\right) .{ }^{13} \mathrm{C} \mathrm{NMR}\left(100.7 \mathrm{MHz}, \mathrm{CD}_{3} \mathrm{OD}\right): \delta$ $153.19,136.95,131.20,131.14,130.96,130.13,129.15,124.32,120.51,116.45,49.33$, 
45.83, 43.59, 31.74, 30.48, 23.38. MALDI-MS: calcd for $(\mathrm{M}+\mathrm{H})^{+} \mathrm{C}_{18} \mathrm{H}_{26} \mathrm{NO}_{4} \mathrm{~S} 380.2$, found $380.4(\mathrm{M}+\mathrm{H})^{+}$.

\section{$N$-9-Fluorenylmethyloxycarbonyl-S-tert-butylthio-L-cysteyl- $N^{(\varepsilon)}$-5-dimethylamino-} naphthalene-1-sulfonyl-L-lysine (Fmoc-Cys $\left(\mathbf{S}^{t} \mathrm{Bu}\right)$-Lys(dans)-OH) (7). At $0^{\circ} \mathrm{C}$, to a solution of Fmoc-Cys $\left(\mathrm{S}^{t} \mathrm{Bu}\right)-\mathrm{OH} 6(1.000 \mathrm{~g}, 2.32 \mathrm{mmol})$ and $\mathrm{N}$-hydroxysuccinimide $(0.267 \mathrm{~g}, 2.32$ $\mathrm{mmol})$ in dry THF (25 $\mathrm{mL}$ ) under an argon atmosphere was added dicyclohexylcarbodiimide $(0.502 \mathrm{~g}, 2.43 \mathrm{mmol})$. The mixture was left stirring to warm to room temperature for $18 \mathrm{~h}$, cooled to $0^{\circ} \mathrm{C}$ and filtered over Celite ${ }^{\circledR}$. The solvent was removed under reduced pressure to yield the crude product which was used without further purification.

At $0^{\circ} \mathrm{C}$, to a solution of $\mathrm{H}$-Lys(dansyl)-OH $5(100 \mathrm{mg}, 0.26 \mathrm{mmol})$ in dry dichloromethane/methanol 1:1 $(16 \mathrm{~mL})$ was added dry triethylamine $(74 \mu \mathrm{l}, 0.52 \mathrm{mmol})$ and a solution of Fmoc-Cys $\left(\mathrm{S}^{t} \mathrm{Bu}\right)-\mathrm{OSu}(178 \mathrm{mg}, 0.34 \mathrm{mmol})$ in dry dichloromethane $(2 \mathrm{~mL})$. The mixture was left to stir for $45 \mathrm{~min}$ at $0^{\circ} \mathrm{C}$ and for $2.5 \mathrm{~h}$ at room temperature, and $0.5 \mathrm{~N}$ $\mathrm{NaHCO}_{3}(30 \mathrm{~mL})$ and dichloromethane $(50 \mathrm{~mL})$ were added. The aqueous layer was extracted with dichloromethane $(3 \times 40 \mathrm{~mL})$, and the combined organic layers were dried over $\mathrm{Na}_{2} \mathrm{SO}_{4}$, filtered, and the solvent was removed under reduced pressure. Purification on silica gel by flash chromatography with ethyl acetate/methanol 40:1, followed by 10:1 as eluent yielded 199 $\mathrm{mg}(95 \%)$ of the desired product 7 as a yellowish-green solid. $R_{f}=0.33$ (dichloromethane/methanol 10:1). $\mathrm{mp}=116^{\circ} \mathrm{C} .[\alpha]^{20}{ }_{\mathrm{D}}=-12.9\left(c=1.0, \mathrm{CHCl}_{3}\right) .{ }^{1} \mathrm{H}$ NMR $(400$ $\left.\mathrm{MHz}, \mathrm{CDCl}_{3} / \mathrm{CD}_{3} \mathrm{OD} 10: 1\right): \delta 8.51(\mathrm{~d}, J=8.8 \mathrm{~Hz}, 1 \mathrm{H}, \mathrm{CH}$ dansyl), $8.32(\mathrm{~d}, J=8.3 \mathrm{~Hz}, 1 \mathrm{H}, \mathrm{CH}$ dansyl), 8.18 (dd, $J=7.4,1.0 \mathrm{~Hz}, 1 \mathrm{H}, \mathrm{CH}$ dansyl), 7.75 (d, $J=7.2 \mathrm{~Hz}, 2 \mathrm{H}, \mathrm{CH}$ Fmoc), 7.62 (d, $J=7.6 \mathrm{~Hz}, 2 \mathrm{H}, \mathrm{CH}$ Fmoc), $7.54(\mathrm{dd}, J=8.2 \mathrm{~Hz}, 1 \mathrm{H}, \mathrm{CH}$ dansyl), $7.49(\mathrm{dd}, J=8.4,7.6 \mathrm{~Hz}, 1 \mathrm{H}$, CH dansyl), 7.38 (dd, $J=7.4 \mathrm{~Hz}, 2 \mathrm{H}, \mathrm{CH}$ Fmoc), 7.28 (dd, $J=7.4 \mathrm{~Hz}, 2 \mathrm{H}, \mathrm{CH}$ Fmoc), 7.18 (d, $J=7.2 \mathrm{~Hz}, 1 \mathrm{H}, \mathrm{CH}$ dansyl), 4.48-4.51 (m, $1 \mathrm{H}, \alpha-\mathrm{CH}), 4.40-4.45\left(\mathrm{~m}, 2 \mathrm{H}, \mathrm{OCH}_{2}\right), 4.31-4.36(\mathrm{~m}$, $1 \mathrm{H}, \alpha-\mathrm{CH}$ ), $4.23\left(\mathrm{t}, J=7.0 \mathrm{~Hz}, 1 \mathrm{H}, \mathrm{CH}_{\mathbf{H}} \mathrm{H}_{2} \mathrm{O}\right.$ ), 3.15 (dd, $\left.J=13.8,5.4 \mathrm{~Hz}, 1 \mathrm{H}, \beta-\mathrm{CH}_{2 \mathrm{a}} \mathrm{Cys}\right), 3.04$ (dd, $J=12.6,8.6 \mathrm{~Hz}, 1 \mathrm{H}, \beta-\mathrm{CH}_{2 b}$ Cys), $2.87\left(\mathrm{~s}, 6 \mathrm{H}, \mathrm{N}\left(\mathrm{CH}_{3}\right)_{2}\right), 2.83-2.88\left(\mathrm{~m}, 2 \mathrm{H}, \varepsilon-\mathrm{CH}_{2}\right), 1.73-$ $1.81\left(\mathrm{~m}, 1 \mathrm{H}, \beta-\mathrm{CH}_{2 \mathrm{a}}\right.$ Lys), 1.56-1.65 (m, $\left.1 \mathrm{H}, \beta-\mathrm{CH}_{2 \mathrm{~b}} \mathrm{Lys}\right), 1.33\left(\mathrm{~s}, 9 \mathrm{H}, \mathrm{C}\left(\mathrm{CH}_{3}\right)_{3}\right), 1.26-1.45(\mathrm{~m}$, $\left.4 \mathrm{H}, \delta-, \gamma-\mathrm{CH}_{2}\right) .{ }^{13} \mathrm{C}$ NMR $\left(100.7 \mathrm{MHz}, \mathrm{CDCl}_{3}\right): \delta 174.53,170.56,156.41,151.30,143.70$, $143.58,141.15,135.01,130.06,129.68,129.56,129.27,128.23,127.65,127.09,125.26$, 123.27, 119.85, 119.25, 115.32, 67.60, 54.73, 52.11, 48.31, 46.91, 45.38, 42.56, 42.08, 31.27, 29.79, 28.44, 21.66. HRMS (FAB; 3-NBA) $\mathrm{m} / \mathrm{z}$ : calcd for $(\mathrm{M}+\mathrm{H})^{+} \mathrm{C}_{40} \mathrm{H}_{49} \mathrm{~N}_{4} \mathrm{O}_{7} \mathrm{~S}_{3} 793.2763$, found 793.2778 .

(E,E,E)-Geranylgeranylchloride (GGCI) (9). To a solution of $N$-chlorosuccinimide (253 mg, $1.89 \mathrm{mmol}$ ), recrystallized from water, in dry $\mathrm{CH}_{2} \mathrm{Cl}_{2}(4 \mathrm{~mL})$, stirring at $-40^{\circ} \mathrm{C}$ under an argon atmosphere, was added dropwise dimethyl sulfide $(150 \mu \mathrm{L}, 2.07 \mathrm{mmol})$. The mixture was then warmed to $0^{\circ} \mathrm{C}$ with an ice bath and kept at this temperature for $5 \mathrm{~min}$. The mixture was cooled to $-40^{\circ} \mathrm{C}$, and a solution of $(E, E, E)$-geranylgeraniol $(500 \mathrm{mg}, 1.72 \mathrm{mmol})$ in $\mathrm{CH}_{2} \mathrm{Cl}_{2}$ (2 $\mathrm{mL}$ ) was added dropwise. The resulting milky white solution was warmed to $-20^{\circ} \mathrm{C}$ and then left stirring to warm to $0^{\circ} \mathrm{C}$ over $2 \mathrm{~h}$. The resulting clear yellow solution was poured into a separating funnel containing dichloromethane $(30 \mathrm{~mL})$ and ice cold brine solution $(20 \mathrm{~mL})$. The layers were separated, and the organic layer was washed with ice cold brine solution $(3 \times 30$ $\mathrm{mL}$ ), dried over $\mathrm{Na}_{2} \mathrm{SO}_{4}$ and filtered. The solvent was removed under reduced pressure, to yield the allylic chloride 9 as a pale yellowish oil (504 $\mathrm{mg}(95 \%)) . R_{f}=0.73$ (cyclohexane/ethyl acetate 10:1). ${ }^{1} \mathrm{H}$ NMR $\left(400 \mathrm{MHz}, \mathrm{CDCl}_{3}\right): \delta 5.45$ (tq, $\left.J=7.8,1.3 \mathrm{~Hz}, 1 \mathrm{H}, \mathrm{CH}_{-}-\mathrm{CH}_{2}-\mathrm{Cl}\right), 5.07-$ $5.13(\mathrm{~m}, 3 \mathrm{H}, \mathrm{CH}), 4.10\left(\mathrm{~d}, J=7.6 \mathrm{~Hz}, 1 \mathrm{H}, \mathrm{CH}_{2}-\mathrm{Cl}\right), 2.03-2.15\left(\mathrm{~m}, 8 \mathrm{H}, 4{ }^{*} \mathrm{CH}_{2}\right), 1.94-2.01(\mathrm{~m}$, $4 \mathrm{H}, 2$ * $\mathrm{CH}_{2}$ ), $1.73\left(\mathrm{~d}, J=1.2 \mathrm{~Hz}, 3 \mathrm{H}, \mathrm{CH}_{3}\right), 1.68\left(\mathrm{~d}, J=0.8 \mathrm{~Hz}, 3 \mathrm{H}, \mathrm{CH}_{3}\right), 1.60(\mathrm{~s}, 9 \mathrm{H}, 3$ * $\left.\mathrm{CH}_{3}\right) .{ }^{13} \mathrm{C}$ NMR $\left(100.7 \mathrm{MHz}, \mathrm{CDCl}_{3}\right): \delta 142.77,135.61,134.96,131.25,124.37,124.16$, 
$123.45,120.29,41.15,39.72,39.67,39.45,26.77,26.61,26.13,25.69,17.68,16.11,16.05$, 16.00 .

S-Geranylgeranyl-L-cysteine methyl ester (H-Cys(GG)-OMe) (10a). At $0^{\circ} \mathrm{C}, \mathrm{HCl}$ * $\mathrm{H}$-Cys-OMe 8a (325 mg, $1.89 \mathrm{mmol})$ was dissolved in $2 \mathrm{~N} \mathrm{NH}_{3} /$ methanol $(12 \mathrm{~mL})$ under an argon atmosphere. The solution was cooled to $-20^{\circ} \mathrm{C}$ and a solution of $(E, E, E)$ geranylgeranylchloride $(9)(531 \mathrm{mg}, 1.72 \mathrm{mmol})$ in dry THF $(2 \mathrm{~mL})$ was added dropwise. The mixture was left stirring to warm to $0^{\circ} \mathrm{C}$ in $2 \mathrm{~h}$. The solvent was removed by coevaporation with toluene $(2 \times 50 \mathrm{~mL})$. Purification on silica gel by flash chromatography with cyclohexane/ethyl acetate $6: 1$ as eluent yielded $470 \mathrm{mg}(67 \%)$ of the desired product 10a as a colorless oil. $R_{f}=0.3$ (cyclohexane/ethyl acetate 1:1). ${ }^{1} \mathrm{H}$ NMR $\left(400 \mathrm{MHz}, \mathrm{CDCl}_{3}\right): \delta 5.24$ (tq, $J$ $\left.=7.8,1.1 \mathrm{~Hz}, 1 \mathrm{H}, \mathrm{C}=\mathrm{C} \underline{\mathrm{H}}-\mathrm{CH}_{2}-\mathrm{S} \mathrm{GG}\right), 5.08-5.12(\mathrm{~m}, 3 \mathrm{H}, \mathrm{CH} \mathrm{GG}), 3.74\left(\mathrm{~s}, 3 \mathrm{H}, \mathrm{OCH}_{3}\right), 3.63$ (dd, $J=7.8,4.4 \mathrm{~Hz}, 1 \mathrm{H}, \alpha-\mathrm{CH}$ ), 3.21 (dd, $J=13.4,7.8 \mathrm{~Hz}, 1 \mathrm{H}, \mathrm{CH}_{2 \mathrm{a}}-\mathrm{S} \mathrm{GG}$ ), 3.16 (dd, $J=13.2,7.6$ $\mathrm{Hz}, 1 \mathrm{H}, \mathrm{CH}_{2 b}-\mathrm{S} \mathrm{GG}$ ), $2.88\left(\mathrm{dd}, J=13.6,4.8 \mathrm{~Hz}, 1 \mathrm{H}, \beta-\mathrm{CH}_{2 \mathrm{a}}\right), 2.68(\mathrm{dd}, J=13.6,7.6 \mathrm{~Hz}, 1 \mathrm{H}, \beta-$ $\left.\mathrm{CH}_{2 \mathrm{~b}}\right), 2.05-2.13\left(\mathrm{~m}, 8 \mathrm{H}, 4{ }^{*} \mathrm{CH}_{2}\right), 1.95-2.00\left(\mathrm{~m}, 4 \mathrm{H}, 2{ }^{*} \mathrm{CH}_{2}\right), 1.70\left(\mathrm{br}, 2 \mathrm{H}, \mathrm{NH}_{2}\right), 1.68(\mathrm{~d}, J=$ $\left.0.8 \mathrm{~Hz}, 3 \mathrm{H}, \mathrm{CH}_{3}\right), 1.67\left(\mathrm{~d}, \mathrm{~J}=0.8 \mathrm{~Hz}, 3 \mathrm{H}, \mathrm{CH}_{3}\right), 1.60\left(\mathrm{~s}, 9 \mathrm{H}, 3{ }^{*} \mathrm{CH}_{3}\right) \cdot{ }^{13} \mathrm{C} \mathrm{NMR}(100.7 \mathrm{MHz}$, $\left.\mathrm{CDCl}_{3}\right): \delta 174.47,139.49,135.30,134.87,131.15,124.32,124.12,123.69,119.90,54.24$, 52.07, 39.66, 39.63, 39.58, 36.39, 29.79, 26.71, 26.57, 26.38, 25.62, 17.61, 16.07, 15.97, 15.94. HRMS (FAB; glycerol) $\mathrm{m} / z$ : calcd for $(\mathrm{M}+\mathrm{H})^{+} \mathrm{C}_{24} \mathrm{H}_{42} \mathrm{NO}_{2} \mathrm{~S} 408.2936$, found 408.2961.

S-Geranylgeranyl-L-cysteine (H-Cys(GG)-OH) (10b). At $0^{\circ} \mathrm{C}, \mathrm{HCl}^{*} \mathrm{H}-\mathrm{Cys}-\mathrm{OH}$ 8b $(271$ $\mathrm{mg}, 1.72 \mathrm{mmol}$ ) was dissolved in $2 \mathrm{~N} \mathrm{NH}_{3} /$ methanol $(10 \mathrm{~mL})$ under an argon atmosphere. A solution of $(E, E, E)$-geranylgeranylchloride (9) $(531 \mathrm{mg}, 1.72 \mathrm{mmol})$ dry THF $(2 \mathrm{~mL})$ was added dropwise. The mixture was left stirring for $3.5 \mathrm{~h}$ at $0^{\circ} \mathrm{C}$ and then for $1.5 \mathrm{~h}$ at room temperature. The solvent was removed by coevaporation with toluene $(80 \mathrm{~mL})$. The compound was purified by flash chromatography on silica gel using a gradient elution starting with dichloromethane/methanol $15: 1$, followed by $10: 1$, then $8: 1$, and finally $5: 1$. After purification $591 \mathrm{mg}(87 \%)$ of the desired product $10 \mathrm{~b}$ were obtained as a white solid. $R_{f}=0.18$ (dichloromethane/methanol 7:1). $\mathrm{mp}=163-166^{\circ} \mathrm{C} .[\alpha]^{20} \mathrm{D}=-12.0\left(c=0.2, \mathrm{CH}_{3} \mathrm{OH}\right) .{ }^{1} \mathrm{H}$ NMR (400 MHz, CD ${ }_{3} \mathrm{OD}$ ): $\delta 5.21$ (tq, $J=7.8,1.1 \mathrm{~Hz}, 1 \mathrm{H}, \mathrm{C}=\mathrm{CH}-\mathrm{CH}_{2}-\mathrm{S} \mathrm{GG}$ ), 5.00-5.07 (m, 3H, 3 * CH GG), 3.61 (dd, $J=8.8,3.6 \mathrm{~Hz}, 1 \mathrm{H}, \alpha-\mathrm{CH}), 3.14-3.25\left(\mathrm{~m}, 1 \mathrm{H}, \mathrm{CH}_{2 \mathrm{a}}-\mathrm{S} \mathrm{GG}\right), 3.17$ (dd, $J=$ 13.0, 7.8 Hz, 1H, $\mathrm{CH}_{2 b}-\mathrm{S} \mathrm{GG}$ ), 3.07 (dd, $J=14.8,3.6 \mathrm{~Hz}, 1 \mathrm{H}, \beta-\mathrm{CH}_{2 \mathrm{a}}$ ), 2.77 (dd, $J=14.6,9.0$ $\mathrm{Hz}, 1 \mathrm{H}, \beta-\mathrm{CH}_{2 \mathrm{~b}}$ ), 1.88-2.08 (m, $12 \mathrm{H}, 6{ }^{*} \mathrm{CH}_{2} \mathrm{GG}$ ), 1.64 (s, 3H, $\left.\mathrm{CH}_{3}\right), 1.59$ (d, J=1.2 Hz, 3H, $\left.\mathrm{CH}_{3}\right), 1.54\left(\mathrm{~s}, 3 \mathrm{H}, \mathrm{CH}_{3}\right), 1.52\left(\mathrm{~s}, 6 \mathrm{H}, 2{ }^{*} \mathrm{CH}_{3}\right) \cdot{ }^{13} \mathrm{C} \mathrm{NMR}\left(100.7 \mathrm{MHz}, \mathrm{CDCl}_{3}\right): \delta$ 173.31, $140.98,136.28,135.91,132.10,125.51,125.21,121.12,55.39,40.91,40.84,40.81,34.08$, $30.32,27.87,27.66,27.54,25.94,17.80,16.34,16.16$. HRMS (FAB; glycerol) $\mathrm{m} / \mathrm{z}$ : calcd for $(\mathrm{M}+\mathrm{H})^{+} \mathrm{C}_{23} \mathrm{H}_{40} \mathrm{NO}_{2} \mathrm{~S} 394.2780$, found 394.2809.

N-tert-Butyloxycarbonyl-S-tert-butylthio-L-cysteyl-L-serine ally lester (Boc-Cys( $\left.{ }^{t} B u\right)-$ Ser-OAll) (13). This compound was prepared by means of the standard peptide coupling procedure with the protected cysteine $11(0.504 \mathrm{~g}, 1.63 \mathrm{mmol})$, the serine allyl ester $12(0.500$ $\mathrm{g}, 1.63 \mathrm{mmol})$ and dry triethylamine $(0.26 \mathrm{~mL}, 1.87 \mathrm{mmol})$. Purification by flash chromatography on silica gel with cyclohexane/ethyl acetate $2: 1$, followed by $1: 1$ as eluent yielded $0.390 \mathrm{~g}(55 \%)$ of the desired product 13 as a colorless oil. $R_{f}=0.22$ (cyclohexane/ethyl acetate 1:1). $[\alpha]^{20}{ }_{\mathrm{D}}=-28.7\left(c=1.0, \mathrm{CHCl}_{3}\right) .{ }^{1} \mathrm{H}$ NMR $\left(400 \mathrm{MHz}, \mathrm{CDCl}_{3}\right): \delta 7.13(\mathrm{~d}, J=7.2 \mathrm{~Hz}$, $1 \mathrm{H}, \mathrm{NH}$ ), 5.91 (ddt, $J=17.2,10.8,5.7 \mathrm{~Hz}, 1 \mathrm{H}, \mathrm{C} \underline{\mathrm{H}}=\mathrm{CH}_{2}$ ), 5.41 (d, $J=4.8,1 \mathrm{H}, \mathrm{NH}$ ), 5.35 (ddt, $J$ $\left.=17.0,1.5,1.4 \mathrm{~Hz}, 1 \mathrm{H}, \mathrm{CH}=\mathrm{C}_{2 a}\right), 5.27\left(\mathrm{dd}, J=10.6,1.4 \mathrm{~Hz}, 1 \mathrm{H}, \mathrm{CH}=\mathrm{CH}_{2 \mathrm{~b}}\right), 4.69(\mathrm{~d}, J=5.6$ $\mathrm{Hz}, 2 \mathrm{H}, \mathrm{OCH}_{2} \mathrm{OAll}$ ), 4.64 (ddd, $J=6.8,3.4 \mathrm{~Hz}, 1 \mathrm{H}, \alpha-\mathrm{CH}$ ), 4.35 (ddd, $J=6.3,5.6 \mathrm{~Hz}, 1 \mathrm{H}, \alpha-$ $\mathrm{CH}$ ), 4.05 (dd, $J=11.6,2.4 \mathrm{~Hz}, 1 \mathrm{H}, \beta-\mathrm{CH}_{2 \mathrm{a}} \operatorname{Ser}$ ), 3.95 (dd, $J=11.6,3.6 \mathrm{~Hz}, 1 \mathrm{H}, \beta-\mathrm{CH}_{2 b}$ Ser), 
3.15 (dd, $J=14.0,7.2 \mathrm{~Hz}, 1 \mathrm{H}, \beta-\mathrm{CH}_{2 \mathrm{a}}$ Cys), 3.09 (dd, $J=13.8,5.4 \mathrm{~Hz}, 1 \mathrm{H}, \beta-\mathrm{CH}_{2 b}$ Cys), 1.46 (s, $\left.9 \mathrm{H}, \mathrm{C}\left(\mathrm{CH}_{3}\right)_{3} \mathrm{Boc}\right), 1.34\left(\mathrm{~s}, 9 \mathrm{H}, \mathrm{C}\left(\mathrm{CH}_{3}\right)_{3} \mathrm{~S}^{t} \mathrm{Bu}\right) .{ }^{13} \mathrm{C} \mathrm{NMR}\left(100.7 \mathrm{MHz}, \mathrm{CDCl}_{3}\right): \delta 170.63$, 169.73, 155.64, 131.31, 118.84, 80.70, 66.27, 62.59, 55.06, 54.39, 48.27, 42.03, 29.76, 28.23. HRMS (FAB; 3-NBA) $\mathrm{m} / \mathrm{z}$ : calcd for $(\mathrm{M}+\mathrm{H})^{+} \mathrm{C}_{18} \mathrm{H}_{33} \mathrm{~N}_{2} \mathrm{O}_{6} \mathrm{~S}_{2}$ 437.1780, found 437.1795.

\section{L-Cysteyl-S-tert-butylthio-L-serine allyl ester-hydrotrifluoracetate (TFA*H-Cys( $\left.{ }^{t} B u\right)-$} Ser-OAll) (14). This compound was prepared by means of the standard Boc-removal procedure with the dipeptide Boc-Cys $\left(\mathrm{S}^{t} \mathrm{Bu}\right)$-Ser-OAll $13(0.665 \mathrm{~g}, 1.52 \mathrm{mmol})$ without addition of thioanisole. The compound was purified by flash chromatography on silica gel using a gradient elution starting with dichloromethane/methanol $50: 1$, followed by $30: 1$, and finally 10:1. After purification $0.624 \mathrm{~g}(91 \%)$ of the desired product 14 were obtained as a colorless oil. $R_{f}=0.30$ (dichloromethane/methanol 10:1). $[\alpha]^{20}{ }_{\mathrm{D}}=+12.2\left(c=1.0, \mathrm{CHCl}_{3}\right) .{ }^{1} \mathrm{H}$ NMR $(400$ $\mathrm{MHz}, \mathrm{CDCl}_{3} / \mathrm{CD}_{3} \mathrm{OD} 10: 1$ ): $\delta 5.94$ (ddt, $J=17.2,10.4,5.7 \mathrm{~Hz}, 1 \mathrm{H}, \mathrm{C} \underline{\mathrm{H}}=\mathrm{CH}_{2}$ ), 5.36 (ddt, $J=$ $17.4,1.5,1.4 \mathrm{~Hz}, 1 \mathrm{H}, \mathrm{CH}=\underline{\mathrm{C}}_{2 \mathrm{a}}$ ), 5.27 (ddt, $J=10.6,1.4 \mathrm{~Hz}, 1 \mathrm{H}, \mathrm{CH}=\underline{\mathrm{C}}_{2 \mathrm{~b}}$ ), 4.69 (ddt, $J=5.6$, 1.3, $1.2 \mathrm{~Hz}, 2 \mathrm{H}, \mathrm{OCH}_{2} \mathrm{OAll}$ ), 4.59 (dd, $J=3.6 \mathrm{~Hz}, 1 \mathrm{H}, \alpha-\mathrm{CH}$ Ser), 3.99 (dd, $J=11.2,4.0 \mathrm{~Hz}$, $1 \mathrm{H}, \beta-\mathrm{CH}_{2 \mathrm{a}}$ Ser), 3.87 (dd, $J=11.6,3.6 \mathrm{~Hz}, 1 \mathrm{H}, \beta-\mathrm{CH}_{2 \mathrm{~b}}$ Ser), 3.71 (dd, $J=8.8,4.0 \mathrm{~Hz}, 1 \mathrm{H}, \alpha-$ CH Cys), 3.20 (dd, $J=13.6,4.0 \mathrm{~Hz}, 1 \mathrm{H}, \beta-\mathrm{CH}_{2 \mathrm{a}}$ Cys), 2.80 (dd, $J=13.6,8.8 \mathrm{~Hz}, 1 \mathrm{H}, \beta-\mathrm{CH}_{2 b}$ Cys), $1.36\left(\mathrm{~s}, 9 \mathrm{H}, \mathrm{C}\left(\mathrm{CH}_{3}\right)_{3}\right) \cdot{ }^{13} \mathrm{C}$ NMR $\left(100.7 \mathrm{MHz}, \mathrm{CDCl}_{3}\right): \delta 173.90,169.76,131.28,119.03$, 66.38, 63.13, 54.98, 54.22, 48.49, 44.93, 29.84. HRMS (FAB; 3-NBA) $\mathrm{m} / \mathrm{z}$ : calcd for (M - TFA $+\mathrm{H})^{+} \mathrm{C}_{13} \mathrm{H}_{25} \mathrm{~N}_{2} \mathrm{O}_{4} \mathrm{~S}_{2} 337.1256$, found 337.1243.

N-9-Fluorenylmethyloxycarbonyl-L-seryl-S-tert-butylthio-L-cysteyl-L-serine allyl ester (Fmoc-Ser-Cys( $\mathbf{S}^{t} \mathrm{Bu}$ )-Ser-OAll) (16). This compound was prepared by means of the standard peptide coupling procedure with Fmoc-Ser-OH $15(251 \mathrm{mg}, 0.77 \mathrm{mmol})$ and the dipeptide H-Cys( $\left.\mathrm{S}^{t} \mathrm{Bu}\right)$-Ser-OAll $14(0.250 \mathrm{~g}, 0.77 \mathrm{mmol})$. Purification by flash chromatography on silica gel with dichloromethane/methanol $40: 1$ as eluent yielded $324 \mathrm{mg}(65 \%)$ of the desired product 16 as a white solid. $R_{f}=0.47$ (dichloromethane/methanol $10: 1$ ). $\mathrm{mp}=157^{\circ} \mathrm{C}$. $t_{R}=16.51 \mathrm{~min}$ (The gradient was changed for: $A / B: 80 / 20(0 \mathrm{~min}), 0 / 100(30 \mathrm{~min}), 0 / 100$ (40 $\min ).)[\alpha]^{20}{ }_{D}=-68.2(c=1.0, \mathrm{DMF}) .{ }^{1} \mathrm{H}$ NMR $\left(400 \mathrm{MHz}, \mathrm{CDCl}_{3} / \mathrm{CD}_{3} \mathrm{OD} 3: 1\right): \delta 7.78(\mathrm{~d}, J=7.6$ $\mathrm{Hz}, 2 \mathrm{H}, \mathrm{CH}$ Fmoc), 7.64-7.67 (m, 2H, CH Fmoc), 7.40 (dd, $J=7.2 \mathrm{~Hz}, 2 \mathrm{H}, \mathrm{CH}$ Fmoc), 7.32 (ddd, $J=7.5,1.1 \mathrm{~Hz}, 2 \mathrm{H}, \mathrm{CH}$ Fmoc), 5.93 (ddt, $J=17.0,10.6,5.7 \mathrm{~Hz}, 1 \mathrm{H}, \mathrm{C} \underline{\mathrm{H}}=\mathrm{CH}_{2}$ ), 5.34 (dd, $\left.J=17.2,1.6 \mathrm{~Hz}, 1 \mathrm{H}, \mathrm{CH}=\underline{\mathrm{C}}_{2 \mathrm{a}}\right), 5.24\left(\mathrm{dd}, J=10.8,1.3 \mathrm{~Hz}, 1 \mathrm{H}, \mathrm{CH}=\mathrm{CH}_{2 b}\right), 4.76(\mathrm{dd}, J=8.4$, $4.8 \mathrm{~Hz}, 1 \mathrm{H}, \alpha-\mathrm{CH}$ Cys), $4.66\left(\mathrm{~d}, J=5.6 \mathrm{~Hz}, 2 \mathrm{H}, \mathrm{OCH}_{2} \mathrm{OAll}\right), 4.58$ (dd, $J=4.2 \mathrm{~Hz}, 1 \mathrm{H}, \alpha-\mathrm{CH}$ Ser), 4.40 (d, $J=6.8 \mathrm{~Hz}, 2 \mathrm{H}, \mathrm{OCH}_{2} \mathrm{Fmoc}$ ), 4.28 (dd, $J=5.6 \mathrm{~Hz}, 1 \mathrm{H}, \alpha-\mathrm{CH}$ Ser'), 4.24 (t, $J=$ $6.8 \mathrm{~Hz}, 1 \mathrm{H}, \mathrm{CHCH}_{2} \mathrm{O} F \mathrm{Foc}$ ), $3.93\left(\mathrm{dd}, J=11.4,4.6 \mathrm{~Hz}, 1 \mathrm{H}, \beta-\mathrm{CH}_{2 \mathrm{a}} \mathrm{Ser}\right), 3.82-3.88(\mathrm{~m}, 2 \mathrm{H}, \beta-$ $\mathrm{CH}_{2 b}$ Ser, $\beta-\mathrm{CH}_{2 \mathrm{a}}$ Ser'), 3.79 (dd, $J=11.2,6.0 \mathrm{~Hz}, 1 \mathrm{H}, \beta-\mathrm{CH}_{2 \mathrm{~b}}$ Ser'), 3.26 (dd, $J=13.8,5.0 \mathrm{~Hz}$, $\left.1 \mathrm{H}, \beta-\mathrm{CH}_{2 \mathrm{a}} \mathrm{Cys}\right), 3.06$ (dd, $\left.J=13.6,8.8 \mathrm{~Hz}, 1 \mathrm{H}, \beta-\mathrm{CH}_{2 \mathrm{~b}} \mathrm{Cys}\right), 1.33\left(\mathrm{~s}, 9 \mathrm{H}, \mathrm{C}\left(\mathrm{CH}_{3}\right)_{3}\right) .{ }^{13} \mathrm{C} \mathrm{NMR}$ $\left(100.7 \mathrm{MHz}, \mathrm{CDCl}_{3} / \mathrm{CD}_{3} \mathrm{OD} 3: 1\right): \delta 170.88,170.18,169.11,156.47,143.20,143.06,140.63$, 140.61, 130.96, 126.99, 126.37, 124.37, 124.33, 119.14, 117.42, 66.52, 65.35, 61.45, 61.04, $56.18,54.46,52.46,47.21,46.40,40.67,28.73$. HRMS (FAB; 3-NBA) $\mathrm{m} / \mathrm{z}$ : calcd for $(\mathrm{M}+\mathrm{H})^{+}$ $\mathrm{C}_{31} \mathrm{H}_{40} \mathrm{~N}_{3} \mathrm{O}_{8} \mathrm{~S}$ 646.2257, found 646.2272.

N-9-Fluorenylmethyloxycarbonyl-L-seryl-S-tert-butylthio-L-cysteyl-L-serine (Fmoc-SerCys $\left.\left(\mathbf{S}^{t} \mathrm{Bu}\right)-\mathrm{Ser}-\mathrm{OH}\right)(\mathbf{1 7})$. This compound was prepared by means of the standard allyl ester removal procedure with the tripeptide Fmoc-Ser-Cys $\left(\mathrm{S}^{t} \mathrm{Bu}\right)$-Ser-OAll $16(200 \mathrm{mg}, 0.31 \mathrm{mmol})$. After washing with diethylether $(5 \times 10 \mathrm{~mL}) 184 \mathrm{mg}(98 \%)$ of the desired product 17 were obtained as an orange-brown solid. $R_{f}=0.3$ (dichloromethane/methanol $5: 1$ ). $\mathrm{mp}=184^{\circ} \mathrm{C}$. $[\alpha]^{20}{ }_{D}=-73.6\left(c=2.25, \mathrm{CH}_{3} \mathrm{OH}\right) .{ }^{1} \mathrm{H}$ NMR $\left(400 \mathrm{MHz}, \mathrm{CDCl}_{3} / \mathrm{CD}_{3} \mathrm{OD} 1: 1\right): \delta 7.77(\mathrm{~d}, J=7.2 \mathrm{~Hz}$, $2 \mathrm{H}, \mathrm{CH}$ Fmoc), 7.64-7.67 (m, 2H, CH Fmoc), 7.39 (dd, $J=7.2 \mathrm{~Hz}, 2 \mathrm{H}, \mathrm{CH}$ Fmoc), 7.31 (dd, $J$ 
$=7.4 \mathrm{~Hz}, 2 \mathrm{H}, \mathrm{CH}$ Fmoc), 4.75-4.78 (m, 1H, $\alpha-\mathrm{CH}$ Cys), 4.52 (dd, $J=4.0 \mathrm{~Hz}, 1 \mathrm{H}, \alpha-\mathrm{CH}$ Ser), 4.40 (d, $J=6.8 \mathrm{~Hz}, 2 \mathrm{H}, \mathrm{OCH}_{2}$ ), 4.29 (dd, $J=5.6 \mathrm{~Hz}, 1 \mathrm{H}, \alpha-\mathrm{CH}$ Ser'), 4.24 (t, $J=6.8 \mathrm{~Hz}, 1 \mathrm{H}$, $\mathrm{CH} \mathrm{CH}_{2} \mathrm{O}$ Fmoc), 3.93 (dd, $J=11.4,4.6 \mathrm{~Hz}, 1 \mathrm{H}, \beta-\mathrm{CH}_{2 \mathrm{a}} \mathrm{Ser}$ ), 3.84-3.87 (m, $2 \mathrm{H}, \beta-\mathrm{CH}_{2 \mathrm{a}}$ Ser', $\beta$ $\mathrm{CH}_{2 \mathrm{~b}}$ Ser), 3.80 (dd, $J=11.0,5.8 \mathrm{~Hz}, 1 \mathrm{H}, \beta-\mathrm{CH}_{2 \mathrm{~b}}$ Ser'), 3.27 (dd, $J=13.8,4.6 \mathrm{~Hz}, 1 \mathrm{H}, \beta-\mathrm{CH}_{2 \mathrm{a}}$ Cys), 3.06 (dd, $J=13.6,8.4 \mathrm{~Hz}, 1 \mathrm{H}, \beta-\mathrm{CH}_{2 \mathrm{~b}}$ Cys), $1.33\left(\mathrm{~s}, 9 \mathrm{H}, \mathrm{C}\left(\mathrm{CH}_{3}\right)_{3}\right) \cdot{ }^{13} \mathrm{C} \mathrm{NMR}(100.7 \mathrm{MHz}$, $\left.\mathrm{CDCl}_{3} / \mathrm{CD}_{3} \mathrm{OD} 1: 1\right): \delta 172.53,172.46,171.60,157.96,144.71,144.57,142.10,128.45$, 127.84, 125.87, 125.82, 120.59, 67.97, 62.95, 62.60, 57.67, 55.84, 53.99, 49.68, 47.89, 42.18, 30.15. HRMS (FAB; triethanolamine) $\mathrm{m} / \mathrm{z}$ : calcd for $(\mathrm{M}-\mathrm{H})^{-} \mathrm{C}_{28} \mathrm{H}_{34} \mathrm{~N}_{3} \mathrm{O}_{8} \mathrm{~S}_{2} 604.1787$, found 604.1792.

\section{N-9-Fluorenylmethyloxycarbonyl-L-seryl-S-tert-butylthio-L-cysteyl-L-seryl-S- geranylgeranyl-L-cysteine methyl ester (Fmoc-Ser-Cys( $\left.\left.{ }^{t} B u\right)-S e r-C y s(G G)-O M e\right)$ (18).} This compound was prepared by means of the standard peptide coupling procedure with the tripeptide Fmoc-Ser-Cys $\left(\mathrm{S}^{t} \mathrm{Bu}\right)$-Ser-OH (17) $(200 \mathrm{mg}, 0.33 \mathrm{mmol})$ and $\mathrm{H}-\mathrm{Cys}(\mathrm{GG})-\mathrm{OMe}(\mathbf{1 0 a})$ (135 mg, $0.33 \mathrm{mmol}$ ). Instead of $\mathrm{HOBt}$, 3-Hydroxy-3,4-dihydro-4-oxo-1,2,3-benzotriazine (HODhbt) was used, and dichloromethane/trifluoroethanol 7.5:1 $(17 \mathrm{~mL})$ was used as solvent. Purification by flash chromatography on silica gel with dichloromethane/methanol 40:1, followed by $30: 1$ as eluent yielded $293 \mathrm{mg}(89 \%)$ of the desired product 18 as a white solid. $R_{f}$ $=0.57$ (dichloromethane/methanol $10: 1) \cdot \mathrm{mp}=169^{\circ} \mathrm{C} . \mathrm{t}_{\mathrm{R}}=18.39 \mathrm{~min} .[\alpha]^{20}{ }_{\mathrm{D}}=-49.6(c=0.5$, $\mathrm{CHCl}_{3}$ ). ${ }^{1} \mathrm{H}$ NMR $\left(400 \mathrm{MHz}, \mathrm{CDCl}_{3} / \mathrm{CD}_{3} \mathrm{OD} 10: 1\right): \delta 7.77$ (d, $J=7.6 \mathrm{~Hz}, 2 \mathrm{H}, \mathrm{CH}$ Fmoc), 7.637.66 (m, 2H, CH Fmoc), 7.39 (dd, $J=7.4 \mathrm{~Hz}, 2 \mathrm{H}, \mathrm{CH}$ Fmoc), 7.31 (ddd, $J=7.4,0.9 \mathrm{~Hz}, 2 \mathrm{H}$, $\mathrm{CH}$ Fmoc), 5.20 (t, $J=7.2 \mathrm{~Hz}, 1 \mathrm{H}, \mathrm{C}=\mathrm{CH}-\mathrm{CH}_{2}-\mathrm{S} \mathrm{GG}$ ), 5.06-5.15 (m, 3H, $\left.3{ }^{*} \mathrm{CH} \mathrm{GG}\right), 4.86$ (dd, $J=8.4,4.8 \mathrm{~Hz}, 1 \mathrm{H}, \alpha-\mathrm{CH}$ Cys $\left._{\mathrm{stBu}}\right), 4.78-4.80(\mathrm{~m}, 1 \mathrm{H}, \alpha-\mathrm{CH}$ Cys $(\mathrm{GG})), 4.50(\mathrm{dd}, J=5.2 \mathrm{~Hz}$, $1 \mathrm{H}, \alpha-\mathrm{CH}$ Ser), 4.41 (d, $J=6.8 \mathrm{~Hz}, 2 \mathrm{H}, \mathrm{OCH}_{2}$ ), 4.29 (dd, $J=5.6 \mathrm{~Hz}, 1 \mathrm{H}, \alpha-\mathrm{CH}$ Ser'), 4.24 (t, $J$ $\left.=6.8 \mathrm{~Hz}, 1 \mathrm{H}, \mathrm{CH}^{\mathrm{H}} \mathrm{CH}_{2} \mathrm{O} \mathrm{Fmoc}\right), 3.78-3.90\left(\mathrm{~m}, 4 \mathrm{H}, 2{ }^{*} \beta-\mathrm{CH}_{2} \mathrm{Ser}\right), 3.75\left(\mathrm{~s}, 3 \mathrm{H}, \mathrm{OCH}_{3}\right), 3.25$ (dd, $J=14.0,5.2 \mathrm{~Hz}, 1 \mathrm{H}, \beta-\mathrm{CH}_{2 \mathrm{a}} \mathrm{Cys}_{\mathrm{stBu}}$ ), 3.23 (dd, $J=13.6,8.0 \mathrm{~Hz}, 1 \mathrm{H}, \mathrm{CH}_{2 \mathrm{a}}-\mathrm{S} \mathrm{GG}$ ), 3.12 (dd, $J$ $=13.2,7.6 \mathrm{~Hz}, 1 \mathrm{H}, \mathrm{CH}_{2 b}-\mathrm{S} \mathrm{GG}$ ), 3.06 (dd, $J=13.6,8.8 \mathrm{~Hz}, 1 \mathrm{H}, \beta-\mathrm{CH}_{2 b} \mathrm{Cys}_{\mathrm{StB}}$ ), 2.96 (dd, $J=$ 13.6, $5.2 \mathrm{~Hz}, 1 \mathrm{H}, \beta-\mathrm{CH}_{2 \mathrm{a}}$ Cys(GG)), 2.82 (dd, $\left.J=14.0,8.0 \mathrm{~Hz}, 1 \mathrm{H}, \beta-\mathrm{CH}_{2 \mathrm{~b}} \mathrm{Cys}(\mathrm{GG})\right)$, 1.93$2.13\left(\mathrm{~m}, 12 \mathrm{H}, 6{ }^{*} \mathrm{CH}_{2} \mathrm{GG}\right), 1.68\left(\mathrm{~d}, J=0.8 \mathrm{~Hz}, 3 \mathrm{H}, \mathrm{CH}_{3}\right), 1.67\left(\mathrm{~d}, J=0.8 \mathrm{~Hz}, 3 \mathrm{H}, \mathrm{CH}_{3}\right), 1.59$ (s, 9H, $\left.3{ }^{*} \mathrm{CH}_{3}\right), 1.49\left(\mathrm{~s}, 9 \mathrm{H}, \mathrm{C}\left(\mathrm{CH}_{3}\right)_{3}\right) \cdot{ }^{13} \mathrm{C} \mathrm{NMR}\left(100.7 \mathrm{MHz}, \mathrm{CDCl}_{3} / \mathrm{CD}_{3} \mathrm{OD} 10: 1\right): \delta 171.31$, $170.55,170.32,169.86,156.47,143.19,143.04,140.62$, 139.11, 134.45, 134.04, 130.25, $126.98,126.36,124.33,123.63,123.52,123.16,119.14,119.09,66.52,61.47,61.09,56.16$, $54.89,53.00,51.74,51.52,47.90,46.41,40.17,39.98,39.94,32.22,28.75,28.69,25.98$, 25.80, 25.68, 24.50, 16.45, 14.97, 14.85, 14.82. HRMS (FAB; 3-NBA) $\mathrm{m} / \mathrm{z}$ : calcd for $(\mathrm{M}+\mathrm{Na})^{+}$ $\mathrm{C}_{52} \mathrm{H}_{74} \mathrm{~N}_{4} \mathrm{NaO}_{9} \mathrm{~S}_{3}$ 1017.4516, found 1017.4536.

L-Seryl-S-tert-butylthio-L-cysteyl-L-seryl-S-geranylgeranyl-L-cysteine methyl ester (H-Ser-Cys(S $\left.{ }^{t} \mathrm{Bu}\right)$-Ser-Cys(GG)-OMe) (19a). At room temperature, to a solution of Fmoc-SerCys $\left(\mathrm{S}^{t} \mathrm{Bu}\right)$-Ser-Cys $(\mathrm{GG})$-OMe $18(208 \mathrm{mg}, 0.21 \mathrm{mmol})$ in dry dichloromethane $(1.7 \mathrm{~mL})$ under an argon atmosphere was added a solution of dry dichloromethane/diethylamine 1:1 (1.7 mL). The mixture was left to stir for $2.5 \mathrm{~h}$, and the solvent was removed by coevaporation with toluene $(40 \mathrm{~mL})$ and chloroform $(40 \mathrm{~mL})$. The compound was purified by flash chromatography on silica gel using a gradient elution starting with cyclohexane/ethyl acetate 1:1, followed by dichloromethane/methanol 40:1, then 30:1, and finally 20:1. After purification $120 \mathrm{mg}(74 \%)$ of the desired product 19a were obtained as a colorless oil. $R_{f}=0.10$ (dichloromethane/methanol 10:1). $[\alpha]^{20}{ }_{\mathrm{D}}=-76.6\left(c=1.15, \mathrm{CH}_{3} \mathrm{OH}\right) .{ }^{1} \mathrm{H}$ NMR $\left(400 \mathrm{MHz}, \mathrm{CDCl}_{3} / \mathrm{CD}_{3} \mathrm{OD} 10: 1\right): \delta 5.21$ (t, $J=$ $\left.7.6 \mathrm{~Hz}, 1 \mathrm{H}, \mathrm{C}=\mathrm{C} \underline{\mathrm{H}}-\mathrm{CH}_{2}-\mathrm{S} \mathrm{GG}\right), 5.08-5.13\left(\mathrm{~m}, 3 \mathrm{H}, 3{ }^{*} \mathrm{CH} \mathrm{GG}\right), 4.70(\mathrm{dd}, J=8.6,5.0 \mathrm{~Hz}, 1 \mathrm{H}, \alpha-$ $\mathrm{CH}$ Cys $\left._{\mathrm{SAB}}\right), 4.66$ (dd, $J=7.8,5.0 \mathrm{~Hz}, 1 \mathrm{H}, \alpha-\mathrm{CH}$ Cys(GG)), $4.50(\mathrm{dd}, J=4.8 \mathrm{~Hz}, 1 \mathrm{H}, \alpha-\mathrm{CH}$ Ser), $3.89\left(\mathrm{dd}, J=11.6,5.2 \mathrm{~Hz}, 1 \mathrm{H}, \beta-\mathrm{CH}_{2 \mathrm{a}} \mathrm{Ser}\right), 3.76\left(\mathrm{~s}, 3 \mathrm{H}, \mathrm{OCH}_{3}\right), 3.75-3.79(\mathrm{~m}, 2 \mathrm{H}, \beta-$ 
$\mathrm{CH}_{2 \mathrm{~b}}$ Ser, $\beta-\mathrm{CH}_{2 \mathrm{a}}$ Ser'), 3.70 (dd, $J=10.6,6.2 \mathrm{~Hz}, 1 \mathrm{H}, \beta-\mathrm{CH}_{2 \mathrm{~b}}$ Ser'), 3.51 (dd, $J=5.8 \mathrm{~Hz}, 1 \mathrm{H}$, $\alpha$-CH Ser), 3.24 (dd, $J=13.0,8.2 \mathrm{~Hz}, 1 \mathrm{H}, \mathrm{CH}_{2 \mathrm{a}}-\mathrm{S} \mathrm{GG}$ ), 3.23 (dd, $J=13.6,4.8 \mathrm{~Hz}, 1 \mathrm{H}, \beta-\mathrm{CH}_{2 \mathrm{a}}$ Cys ${ }_{\mathrm{StBu}}$ ), 3.13 (dd, $J=13.0,7.4 \mathrm{~Hz}, 1 \mathrm{H}, \mathrm{CH}_{2 b}-\mathrm{S} \mathrm{GG}$ ), 3.07 (dd, $J=13.6,8.4 \mathrm{~Hz}, 1 \mathrm{H}, \beta-\mathrm{CH}_{2 b}$ Cys ${ }_{\mathrm{StBu}}$ ), 2.97 (dd, $J=13.8,5.0 \mathrm{~Hz}, 1 \mathrm{H}, \beta-\mathrm{CH}_{2 \mathrm{a}}$ Cys(GG)), $2.83(\mathrm{dd}, J=13.6,8.0 \mathrm{~Hz}, 1 \mathrm{H}, \beta-$ $\left.\mathrm{CH}_{2 \mathrm{~b}} \mathrm{Cys}(\mathrm{GG})\right), 1.96-2.14\left(\mathrm{~m}, 12 \mathrm{H}, 6{ }^{*} \mathrm{CH}_{2} \mathrm{GG}\right), 1.69\left(\mathrm{~s}, 6 \mathrm{H}, 2\right.$ * $\left.\mathrm{CH}_{3}\right), 1.61\left(\mathrm{~s}, 9 \mathrm{H}, 3{ }^{*} \mathrm{CH}_{3}\right)$, $1.36\left(\mathrm{~s}, 9 \mathrm{H}, \mathrm{C}\left(\mathrm{CH}_{3}\right)_{3}\right) .{ }^{13} \mathrm{C} \mathrm{NMR}\left(100.7 \mathrm{MHz}, \mathrm{CDCl}_{3} / \mathrm{CD}_{3} \mathrm{OD} 10: 1\right): \delta 174.31,170.70,170.28$, 169.89, 139.66, 134.90, 134.46, 130.76, 123.92, 123.74, 123.32, 119.01, 64.12, 61.63, 55.80, $54.90,53.13,52.29,52.00,48.07,41.03,39.48,39.45,32.26,29.50,29.33,26.53,26.40$, $26.29,25.35,17.36,15.81,15.72,15.69$. HRMS (FAB; 3-NBA) $\mathrm{m} / \mathrm{z}$ : calcd for $(\mathrm{M}+\mathrm{Na})^{+}$ $\mathrm{C}_{37} \mathrm{H}_{64} \mathrm{~N}_{4} \mathrm{NaO}_{7} \mathrm{~S}_{3}$ 795.3835, found 795.3852.

N-9-Fluorenylmethyloxycarbonyl-L-seryl-S-geranylgeranyl-L-cysteine (Fmoc-SerCys(GG)-OH) (20). At $0^{\circ} \mathrm{C}$, to a solution of Fmoc-Ser-OH $15(3.000 \mathrm{~g}, 9.17 \mathrm{mmol})$ and $\mathrm{N}$ hydroxysuccinimide $(1.055 \mathrm{~g}, 9.17 \mathrm{mmol})$ in dry THF $(15 \mathrm{~mL})$ under an argon atmosphere was added dicyclohexylcarbodiimide $(1.986 \mathrm{~g}, 9.62 \mathrm{mmol})$. The mixture was left stirring to warm to room temperature for $18 \mathrm{~h}$, cooled to $0^{\circ} \mathrm{C}$ and filtered over Celite ${ }^{\circledR}$. The solvent was removed under reduced pressure to yield the crude product which was used without further purification.

At $0^{\circ} \mathrm{C}$, to a solution of $\mathrm{H}$-Cys(GG)-OH $10 \mathrm{~b}(390 \mathrm{mg}, 0.99 \mathrm{mmol})$ in dry dichloromethane/methanol 2:1 $(60 \mathrm{~mL})$ was added dry triethylamine $(0.15 \mathrm{~mL}, 1.09 \mathrm{mmol})$ and a solution of Fmoc-Ser-OSu (630 mg, $1.49 \mathrm{mmol}$ ) in dry dichloromethane/methanol 10:1 (2 $\mathrm{mL}$ ). The mixture was left to stir for $1.5 \mathrm{~h}$ at $0^{\circ} \mathrm{C}$ and for $2 \mathrm{~h}$ at room temperature. The mixture was then cooled to $0^{\circ} \mathrm{C}$, Fmoc-Ser-OSu $(570 \mathrm{mg}, 1.344 \mathrm{mmol})$ and triethylamine $(0.09 \mathrm{~mL}$, $0.66 \mathrm{mmol}$ ) were added and the resulting mixture was left to stir $6 \mathrm{~h}$ at $0^{\circ} \mathrm{C}$ and $15 \mathrm{~h}$ at room temperature. The solvent was removed by coevaporation with toluene $(40 \mathrm{~mL})$. The crude product was dissolved in diethylether and $0.5 \mathrm{~N} \mathrm{NaHCO}_{3} 1: 1(80 \mathrm{~mL})$. The aqueous layer was extracted with diethylether $(3 \times 40 \mathrm{~mL})$, the combined organic layers were washed with brine (40 $\mathrm{mL}$ ), dried over $\mathrm{Na}_{2} \mathrm{SO}_{4}$, filtered, and the solvent was removed under reduced pressure. Purification on silica gel by flash chromatography with ethyl acetate/methanol $20: 1$, followed by $10: 1$ as eluent yielded $305 \mathrm{mg}(44 \%)$ of the desired product 20 as a white solid. $R_{f}=0.30$ (dichloromethane/methanol 10:1). $\mathrm{mp}=100-101^{\circ} \mathrm{C} .[\alpha]^{20} \mathrm{D}=-25.8\left(c=1.2, \mathrm{CH}_{3} \mathrm{OH}\right) .{ }^{1} \mathrm{H}$ NMR $\left(400 \mathrm{MHz}, \mathrm{CDCl}_{3}\right): \delta 7.77$ (d, J = 7.6 Hz, 2H, CH Fmoc), 7.61-7.64 (m, 2H, CH Fmoc), 7.70 (d, $J=7.2 \mathrm{~Hz}, 1 \mathrm{H}, \mathrm{NH}$ ), 7.40 (dd, $J=7.2 \mathrm{~Hz}, 2 \mathrm{H}, \mathrm{CH}$ Fmoc), 7.32 (dd, $J=7.2 \mathrm{~Hz}, 2 \mathrm{H}, \mathrm{CH}$ Fmoc), $6.38(\mathrm{~d}, J=7.2 \mathrm{~Hz}, 1 \mathrm{H}, \mathrm{NH}), 5.21\left(\mathrm{t}, J=7.4 \mathrm{~Hz}, 1 \mathrm{H}, \mathrm{C}=\mathrm{C} \underline{\mathrm{H}}-\mathrm{CH}_{2}-\mathrm{S} \mathrm{GG}\right), 5.07-5.13(\mathrm{~m}, 3 \mathrm{H}, 3$ * $\mathrm{CH}$ GG), 4.69 (dd, $J=6.6 \mathrm{~Hz}, 4.6,1 \mathrm{H}, \alpha-\mathrm{CH}$ Cys), 4.40 (d, $J=7.2 \mathrm{~Hz}, 2 \mathrm{H}, \mathrm{OCH}_{2}$ ), 4.30 (br, $1 \mathrm{H}, \alpha-\mathrm{CH}$ Ser), 4.24 (t, $J=7.2 \mathrm{~Hz}, 1 \mathrm{H}, \mathrm{CH}_{\mathrm{H}} \mathrm{H}_{2} \mathrm{O} \mathrm{Fmoc}$ ), 3.92 (dd, $J=11.2,4.0 \mathrm{~Hz}, 1 \mathrm{H}, \beta-\mathrm{CH}_{2 \mathrm{a}}$ Ser), 3.71 (dd, $J=11.4,6.2 \mathrm{~Hz}, 1 \mathrm{H}, \beta-\mathrm{CH}_{2 b} \operatorname{Ser}$ ), 3.23 (dd, $J=13.0,8.2 \mathrm{~Hz}, 1 \mathrm{H}, \mathrm{CH}_{2 \mathrm{a}} \mathrm{S} \mathrm{GG}$ ), 3.16 (dd, $J=13.0,7.4 \mathrm{~Hz}, 1 \mathrm{H}, \mathrm{CH}_{2 b}-\mathrm{S} \mathrm{GG}$ ), 3.03 (dd, $J=13.8,4.6 \mathrm{~Hz}, 1 \mathrm{H}, \beta-\mathrm{CH}_{2 \mathrm{a}} \mathrm{Cys}$ ), 2.87 (dd, $\left.J=13.8,7.4 \mathrm{~Hz}, 1 \mathrm{H}, \beta-\mathrm{CH}_{2 b} \mathrm{Cys}\right), 1.96-2.12$ (m, $\left.12 \mathrm{H}, 6{ }^{*} \mathrm{CH}_{2} \mathrm{GG}\right), 1.68\left(\mathrm{~s}, 3 \mathrm{H}, \mathrm{CH}_{3}\right.$ ), $1.66\left(\mathrm{~s}, 3 \mathrm{H}, \mathrm{CH}_{3}\right), 1.59\left(\mathrm{~s}, 9 \mathrm{H}, 3{ }^{*} \mathrm{CH}_{3}\right) .{ }^{13} \mathrm{C} \mathrm{NMR}\left(100.7 \mathrm{MHz}, \mathrm{CDCl}_{3}\right): \delta 172.52,170.77$, $156.40,143.55,143.42,141.00,139.79,135.07,134.65,130.94,127.45,126.82,124.77$, $124.09,123.93,123.51,119.66,119.21,66.99,62.32,55.86,51.96,46.81,39.42,39.39$, $39.36,32.53,29.51,26.46,26.34,26.20,25.26,17.25,15.66,15.59$. HRMS (FAB; triethanolamine) $\mathrm{m} / \mathrm{z}$ : calcd for $(\mathrm{M}-\mathrm{H})^{-} \mathrm{C}_{41} \mathrm{H}_{53} \mathrm{~N}_{2} \mathrm{O}_{6} \mathrm{~S}$ 701.3624, found 701.3635.

$\mathbf{N}^{(\alpha)}$-tert-Butyloxycarbonyl-L-seryl-S-tert-butylthio-L-cysteine methyl ester (Boc-SerCys $\left.\left(\mathbf{S}^{t} \mathrm{Bu}\right)-\mathrm{OMe}\right)$ (23). This compound was prepared by means of the standard peptide coupling procedure with Boc-Ser-OH $22(0.735 \mathrm{~g}, 3.58 \mathrm{mmol})$ and $\mathrm{H}-\mathrm{Cys}\left(\mathrm{S}^{t} \mathrm{Bu}\right)-\mathrm{OMe} 21$ $(0.800 \mathrm{~g}, 3.58 \mathrm{mmol})$. Instead of EDC/HOBt, EEDQ (1.6 equiv.) was used as coupling reagent. The compound was purified by flash chromatography on silica gel using a gradient elution 
starting with cyclohexane/ethyl acetate $2: 1$, then $1: 1$, and finally $1: 2$. After purification $1.352 \mathrm{~g}$ $(92 \%)$ of the desired product 23 were obtained as a colorless oil. $R_{f}=0.30$ (cyclohexane/ethyl acetate 1:1). $[\alpha]^{20} \mathrm{D}=-23.4\left(c=1.0, \mathrm{CHCl}_{3}\right) .{ }^{1} \mathrm{H}$ NMR $\left(400 \mathrm{MHz}, \mathrm{CDCl}_{3}\right): \delta 7.42(\mathrm{~d}, J=4.4 \mathrm{~Hz}$, $1 \mathrm{H}, \mathrm{NH}$ ), 5.60 (d, $J=7.6 \mathrm{~Hz}, 1 \mathrm{H}, \mathrm{NH}$ ), 4.86 (ddd, $J=7.6,6.2,4.6 \mathrm{~Hz}, 1 \mathrm{H}, \alpha-\mathrm{CH}$ Cys), 4.26 (br, $1 \mathrm{H}, \alpha-\mathrm{CH}$ Ser), 4.07 (dd, $J=11.0,2.6 \mathrm{~Hz}, 1 \mathrm{H}, \beta-\mathrm{CH}_{2 \mathrm{a}}$ Ser), $3.78\left(\mathrm{~s}, 3 \mathrm{H}, \mathrm{OCH}_{3}\right), 3.69$ (dd, $J=$ $11.0,5.4 \mathrm{~Hz}, 1 \mathrm{H}, \beta-\mathrm{CH}_{2 \mathrm{~b}} \mathrm{Ser}$ ), 3.22 (dd, $J=14.0,4.8 \mathrm{~Hz}, 1 \mathrm{H}, \beta-\mathrm{CH}_{2 \mathrm{a}}$ Cys), 3.14 (dd, $J=13.8$, $\left.6.2 \mathrm{~Hz}, 1 \mathrm{H}, \beta-\mathrm{CH}_{2 \mathrm{~b}} \mathrm{Cys}\right), 1.47$ (s, 9H, C( $\left.\left.\mathrm{CH}_{3}\right)_{3} \mathrm{Boc}\right), 1.33$ (s, 9H, C( $\left.\left.\mathrm{CH}_{3}\right)_{3} \mathrm{~S}^{t} \mathrm{Bu}\right) .{ }^{13} \mathrm{C} \mathrm{NMR}$ $\left(100.7 \mathrm{MHz}, \mathrm{CDCl}_{3}\right): \delta 171.21,170.72,155.90,80.46,62.97,55.14,52.75,52.18,48.26$, 41.55, 29.71, 28.27. HRMS (FAB; 3-NBA) $\mathrm{m} / \mathrm{z}$ : calcd for $(\mathrm{M}+\mathrm{Na})^{+} \mathrm{C}_{16} \mathrm{H}_{30} \mathrm{~N}_{2} \mathrm{NaO}_{6} \mathrm{~S}_{2}$, 433.1443, found 433.1450 .

L-Seryl-S-tert-butylthio-L-cysteine methyl ester-hydrotrifluoracetate (TFA* $\mathrm{H}$-SerCys $\left.\left(\mathbf{S}^{t} \mathrm{Bu}\right)-\mathrm{OMe}\right)(\mathbf{2 4})$. This compound was prepared by means of the standard Boc-removal procedure from the dipeptide Boc-Ser-Cys $\left(\mathrm{S}^{t} \mathrm{Bu}\right)$-OMe $23(0.800 \mathrm{~g}, 1.95 \mathrm{mmol})$. After washing with diethylether $(2 \times 10 \mathrm{~mL})$ and cyclohexane $(5 \times 10 \mathrm{~mL}), 0.655 \mathrm{~g}(79 \%)$ of the desired product 24 were obtained as a white solid. $R_{f}=0.07$ ( $n$-hexane/ethyl acetate 1:1). $\mathrm{mp}=136^{\circ}$ C. $[\alpha]^{20}{ }_{D}=-74.8\left(c=1.0, \mathrm{CH}_{3} \mathrm{OH}\right) .{ }^{1} \mathrm{H}$ NMR $\left(400 \mathrm{MHz}, \mathrm{CDCl}_{3} / \mathrm{CD}_{3} \mathrm{OD}\right.$ 10:1): $\delta 4.82(\mathrm{dd}, J=$ 8.0, $4.4 \mathrm{~Hz}, 1 \mathrm{H}, \alpha-\mathrm{CH}$ Cys), 4.07 (dd, $J=6.6,4.6 \mathrm{~Hz}, 1 \mathrm{H}, \alpha-\mathrm{CH}$ Ser), 4.01 (dd, $J=12.0,4.4$ $\mathrm{Hz}, 1 \mathrm{H}, \beta-\mathrm{CH}_{2 \mathrm{a}}$ Ser), 3.86 (dd, $J=12.0,6.4 \mathrm{~Hz}, 1 \mathrm{H}, \beta-\mathrm{CH}_{2 \mathrm{~b}}$ Ser), 3.78 (s, 3H, OCH $\mathrm{OCH}_{3}, 3.20$ (dd, $J=14.0,4.8 \mathrm{~Hz}, 1 \mathrm{H}, \beta-\mathrm{CH}_{2 \mathrm{a}} \mathrm{Cys}$ ), 3.07 (dd, $J=13.8,7.8 \mathrm{~Hz}, 1 \mathrm{H}, \beta-\mathrm{CH}_{2 b} \mathrm{Cys}$ ), 1.34 (s, $9 \mathrm{H}$, $\left.\mathrm{C}\left(\mathrm{CH}_{3}\right)_{3}\right) .{ }^{13} \mathrm{C}$ NMR $\left(100.7 \mathrm{MHz}, \mathrm{CDCl}_{3}\right): \delta 170.56,166.98,60.30,54.51,52.39,51.93,47.93$, 40.75, 29.35. HRMS (FAB; 3-NBA) $\mathrm{m} / \mathrm{z}$ : calcd for $(\mathrm{M}-\mathrm{TFA}+\mathrm{H})^{+} \mathrm{C}_{11} \mathrm{H}_{23} \mathrm{~N}_{2} \mathrm{O}_{4} \mathrm{~S}_{2}$ 311.1099, found 311.1093 .

\section{N-9-Fluorenylmethyloxycarbonyl-L-seryl-S-geranylgeranyl-L-cysteyl-L-seryl-S-tert- butylthio-L-cysteine methyl ester (Fmoc-Ser-Cys(GG)-Ser-Cys( $\left.{ }^{t}{ }^{B} \mathrm{Bu}\right)-\mathrm{OMe}$ ) (25). This} compound was prepared by means of the standard peptide coupling procedure with the dipeptide Fmoc-Ser-Cys(GG)-OH 20 (93 mg, 0.13 mmol), the dipeptide TFA*H-SerCys $\left(\mathrm{S}^{t} \mathrm{Bu}\right)$-OMe 24 (56 mg, $\left.0.13 \mathrm{mmol}\right)$ and dry triethylamine $(55 \mu \mathrm{l}, 0.40 \mathrm{mmol})$. Purification on silica gel by flash chromatography with dichloromethane/methanol $40: 1$ as eluent yielded $87 \mathrm{mg}(66 \%)$ of the desired product 25 as a white solid. $R_{f}=0.62$ (dichloromethane/methanol $10: 1) . \mathrm{mp}=174^{\circ} \mathrm{C} . \mathrm{t}_{\mathrm{R}}=18.49 \mathrm{~min} .[\alpha]^{20} \mathrm{D}=-22.6\left(c=0.5, \mathrm{CHCl}_{3}\right) .{ }^{1} \mathrm{H} \mathrm{NMR}(500 \mathrm{MHz}$, $\left.\mathrm{CDCl}_{3} / \mathrm{CD}_{3} \mathrm{OD} 10: 1\right): \delta 7.77$ (d, J = 7.5 Hz, 2H, CH Fmoc), 7.62-7.64 (m, 2H, CH Fmoc), 7.40 (dd, $J=7.8 \mathrm{~Hz}, 2 \mathrm{H}$, CH Fmoc), 7.32 (dd, $J=7.5 \mathrm{~Hz}, 2 \mathrm{H}, \mathrm{CH}$ Fmoc), 5.23 (t, $J=7.5 \mathrm{~Hz}, 1 \mathrm{H}$, $\left.\mathrm{C}=\mathrm{C} \underline{\mathrm{H}}-\mathrm{CH}_{2}-\mathrm{S} \mathrm{GG}\right), 5.07-5.12(\mathrm{~m}, 3 \mathrm{H}, 3$ * $\mathrm{CH} \mathrm{GG}), 4.79(\mathrm{dd}, J=7.0,5.0 \mathrm{~Hz}, 1 \mathrm{H}, \alpha-\mathrm{CH}$ Cys $\left._{\mathrm{StBu}}\right), 4.61$ (dd, $J=7.0,5.0 \mathrm{~Hz}, 1 \mathrm{H}, \alpha-\mathrm{CH}$ Cys(GG)), $4.53(\mathrm{dd}, J=4.8 \mathrm{~Hz}, 1 \mathrm{H}, \alpha-\mathrm{CH}$ Ser), 4.40 (d, $J=7.0 \mathrm{~Hz}, 2 \mathrm{H}, \mathrm{OCH}_{2}$ ), 4.34 (dd, $J=6.0 \mathrm{~Hz}, 1 \mathrm{H}, \alpha-\mathrm{CH}$ Ser'), 4.23 (t, $J=7.0 \mathrm{~Hz}, 1 \mathrm{H}$, $\mathrm{CHCH}_{2} \mathrm{O}$ Fmoc), 3.88-3.92 (m, $2 \mathrm{H}, 2$ * $\left.\beta-\mathrm{CH}_{2 \mathrm{a}} \mathrm{Ser}\right), 3.76\left(\mathrm{~s}, 3 \mathrm{H}, \mathrm{OCH}_{3}\right), 3.74-3.81(\mathrm{~m}, 2 \mathrm{H}, 2$ * $\beta-\mathrm{CH}_{2 b}$ Ser), 3.21 (d, $J=7.5 \mathrm{~Hz}, 2 \mathrm{H}, \mathrm{CH}_{2}-\mathrm{S} \mathrm{GG}$ ), 3.18 (dd, $J=14.0,5.5 \mathrm{~Hz}, 1 \mathrm{H}, \beta-\mathrm{CH}_{2 \mathrm{a}}$ Cys $_{\mathrm{StBu}}$ ), 3.11 (dd, $J=14.0,7.5 \mathrm{~Hz}, 1 \mathrm{H}, \beta-\mathrm{CH}_{2 b} \mathrm{Cys}_{\mathrm{StBu}}$ ), 3.01 (dd, $J=13.8,5.8 \mathrm{~Hz}, 1 \mathrm{H}, \beta-\mathrm{CH}_{2 \mathrm{a}}$ Cys(GG)), 2.87 (dd, $J=13.8,7.8 \mathrm{~Hz}, 1 \mathrm{H}, \beta-\mathrm{CH}_{2 b} \operatorname{Cys}(\mathrm{GG})$ ), 1.96-2.11 (m, $12 \mathrm{H}, 6$ * $\mathrm{CH}_{2} \mathrm{GG}$ ), $1.67\left(\mathrm{~s}, 6 \mathrm{H}, 2{ }^{*} \mathrm{CH}_{3}\right), 1.59\left(\mathrm{~s}, 9 \mathrm{H}, 3{ }^{*} \mathrm{CH}_{3}\right), 1.33\left(\mathrm{~s}, 9 \mathrm{H}, \mathrm{C}\left(\mathrm{CH}_{3}\right)_{3}\right) \cdot{ }^{13} \mathrm{C} \mathrm{NMR}(125.8 \mathrm{MHz}$, $\mathrm{CDCl}_{3} / \mathrm{CD}_{3} \mathrm{OD}$ 10:1): $\delta 171.27,170.69,170.56,170.04,156.38,143.40,143.28,140.90$, $139.79,134.90,134.48,130.77,127.36,126.71,124.65,123.98,123.82,123.41,119.55$, $118.99,66.88,62.07,61.51,55.86,54.95,53.03,52.17,51.75,47.80,46.67,40.91,39.30$, 39.27, 39.24, 32.06, 29.31, 29.25, 26.32, 26.20, 26.08, 25.09, 17.08, 15.57, 15.44, 15.42. HRMS (FAB; 3-NBA) $\mathrm{m} / \mathrm{z}$ : calcd for $(\mathrm{M}+\mathrm{H})^{+} \mathrm{C}_{52} \mathrm{H}_{75} \mathrm{~N}_{4} \mathrm{O}_{9} \mathrm{~S}_{3} 995.4696$, found 995.4675. 
L-Seryl-S-geranylgeranyl-L-cysteyl-L-seryl-S-tert-butylthio-L-cysteine methyl ester (H-Ser-Cys(GG)-Ser-Cys( $\left.{ }^{t} \mathrm{Bu}\right)$-OMe) (19b). At room temperature, to a solution of Fmoc-SerCys(GG)-Ser-Cys $\left(\mathrm{S}^{t} \mathrm{Bu}\right)$-OMe 25 (56 mg, $\left.56 \mu \mathrm{mol}\right)$ in dry dichloromethane $(0.96 \mathrm{~mL})$ under an argon atmosphere was added a solution of dry dichloromethane/diethylamine 1:1 $(0.64 \mathrm{~mL})$. The mixture was left to stir for $3 \mathrm{~h}$, and the solvent was removed by coevaporation with toluene $(40 \mathrm{~mL})$ and chloroform $(40 \mathrm{~mL})$. The compound was purified by flash chromatography on silica gel using a gradient elution starting with cyclohexane/ethyl acetate $1: 1$, followed by dichloromethane/methanol 40:1, then $20: 1$, and finally $10: 1$. After purification $28.6 \mathrm{mg}(66 \%)$ of the desired product $19 \mathrm{~b}$ were obtained as a colorless oil. $R_{f}=0.21$ (dichloromethane/methanol 10:1). $[\alpha]^{20}=-35.0\left(c=0.5, \mathrm{CH}_{2} \mathrm{Cl}_{2} / \mathrm{CH}_{3} \mathrm{OH} 1: 1\right) .{ }^{1} \mathrm{H} \mathrm{NMR}\left(400 \mathrm{MHz}, \mathrm{CDCl}_{3} / \mathrm{CD}_{3} \mathrm{OD} 10: 1\right): \delta$ 5.25 (t $\left.J=7.5 \mathrm{~Hz}, 1 \mathrm{H}, \mathrm{C}=\mathrm{C} \underline{\mathrm{H}}-\mathrm{CH}_{2}-\mathrm{S} \mathrm{GG}\right), 5.08-5.14$ (m, 3H, 3 * $\mathrm{CH} \mathrm{GG}$ ), 4.78 (dd, $J=7.2,4.8$ $\mathrm{Hz}, 1 \mathrm{H}, \alpha-\mathrm{CH}$ Cys $_{\mathrm{stBu}}$ ), 4.56 (dd, $J=7.6,6.0 \mathrm{~Hz}, 1 \mathrm{H}, \alpha-\mathrm{CH}$ Cys(GG)), 4.51 (dd, $J=5.0 \mathrm{~Hz}, 1 \mathrm{H}$, $\alpha$-CH Ser), 3.91 (dd, $\left.J=11.2,5.2 \mathrm{~Hz}, 1 \mathrm{H}, \beta-\mathrm{CH}_{2 \mathrm{a}} \mathrm{Ser}\right), 3.77\left(\mathrm{~s}, 3 \mathrm{H}, \mathrm{OCH}_{3}\right), 3.75-3.81(\mathrm{~m}, 2 \mathrm{H}$, $\beta-\mathrm{CH}_{2 \mathrm{~b}}$ Ser, $\beta-\mathrm{CH}_{2 \mathrm{a}}$ Ser), 3.70 (dd, $J=10.8,6.0 \mathrm{~Hz}, 1 \mathrm{H}, \beta-\mathrm{CH}_{2 \mathrm{~b}}$ Ser), 3.52 (dd, $J=5.2 \mathrm{~Hz}, 1 \mathrm{H}$, $\alpha-\mathrm{CH}_{\text {Ser) }}$ ), 3.24 (d, $J=8.0 \mathrm{~Hz}, 2 \mathrm{H}, \mathrm{CH}_{2}-\mathrm{S} \mathrm{GG}$ ), 3.18 (dd, $J=13.8,5.0 \mathrm{~Hz}, 1 \mathrm{H}, \beta-\mathrm{CH}_{2 \mathrm{a}}$ Cys $_{\mathrm{stBu}}$ ), $3.11\left(\mathrm{dd}, J=13.8,7.4 \mathrm{~Hz}, 1 \mathrm{H}, \beta-\mathrm{CH}_{2 \mathrm{~b}} \mathrm{Cys}_{\mathrm{stBu}}\right), 3.00\left(\mathrm{dd}, J=14.0,6.0 \mathrm{~Hz}, 1 \mathrm{H}, \beta-\mathrm{CH}_{2 \mathrm{a}}\right.$ Cys(GG)), $2.86\left(\mathrm{dd}, J=13.6,7.6 \mathrm{~Hz}, 1 \mathrm{H}, \beta-\mathrm{CH}_{2 b} \mathrm{Cys}(\mathrm{GG})\right), 1.95-2.13\left(\mathrm{~m}, 12 \mathrm{H}, 6{ }^{*} \mathrm{CH}_{2} \mathrm{GG}\right.$ ), $1.69\left(\mathrm{~s}, 3 \mathrm{H}, \mathrm{CH}_{3}\right), 1.68\left(\mathrm{~d}, J=0.8 \mathrm{~Hz}, 3 \mathrm{H}, \mathrm{CH}_{3}\right), 1.60\left(\mathrm{~s}, 9 \mathrm{H}, 3^{*} \mathrm{CH}_{3}\right), 1.34\left(\mathrm{~s}, 9 \mathrm{H}, \mathrm{C}\left(\mathrm{CH}_{3}\right)_{3}\right)$. ${ }^{13} \mathrm{C}$ NMR $\left(100.7 \mathrm{MHz}, \mathrm{CDCl}_{3} / \mathrm{CD}_{3} \mathrm{OD} 10: 1\right): \delta 174.36,170.83,170.65,170.15,139.93,135.07$, $134.63,130.91$, 124.06, 123.90, 123.48, 119.06, 64.05, 61.57, 55.73, 54.88, 52.90, 52.31, $51.85,47.89,41.01,39.40,39.39,39.36,32.46,29.45,29.39,26.43,26.33,26.21,25.23$, 17.22, 15.73, 15.59, 15.56. HRMS (FAB; 3-NBA) $\mathrm{m} / z$ : calcd for $(\mathrm{M}+\mathrm{Na})^{+} \mathrm{C}_{37} \mathrm{H}_{64} \mathrm{~N}_{4} \mathrm{NaO}_{7} \mathrm{~S}_{3}$ 795.3835, found 795.3844 .

$\mathrm{N}$-9-Fluorenylmethyloxycarbonyl-L-seryl-S-geranylgeranyl-L-cysteyl-L-serine allyl ester (Fmoc-Ser-Cys(GG)-Ser-OAll) (26). This compound was prepared by means of the standard peptide coupling procedure with the dipeptide Fmoc-Ser-Cys(GG)-OH 20 (232 mg, $0.33 \mathrm{mmol}, 1$ eq.), TosOH * H-Ser-OAll $12(210 \mathrm{mg}, 0.66 \mathrm{mmol})$ and dry triethylamine (138 $\mu \mathrm{l}$, $0.99 \mathrm{mmol})$. The compound was purified by flash chromatography on silica gel using a gradient elution starting with dichloromethane/methanol 100:1, then 60:1, and finally 30:1. After purification $139 \mathrm{mg} \mathrm{(51 \% )}$ of the desired product 26 were obtained as a white solid. $R_{f}=$ 0.58 (dichloromethane/methanol 10:1). $\mathrm{mp}=157^{\circ} \mathrm{C} . \mathrm{t}_{\mathrm{R}}=15.97 \mathrm{~min} .[\alpha]^{20}{ }_{\mathrm{D}}=-23.4(c=0.5$, $\mathrm{CHCl}_{3} / \mathrm{CH}_{3} \mathrm{OH}$ 3:1). ${ }^{1} \mathrm{H}$ NMR $\left(400 \mathrm{MHz}, \mathrm{CDCl}_{3} / \mathrm{CD}_{3} \mathrm{OD} 10: 1\right): \delta 7.77(\mathrm{~d}, J=7.6 \mathrm{~Hz}, 2 \mathrm{H}, \mathrm{CH}$ Fmoc), 7.60-7.62 (m, 2H, CH Fmoc), 7.41 (dd, $J=7.2 \mathrm{~Hz}, 2 \mathrm{H}, \mathrm{CH}$ Fmoc), 7.32 (ddd, $J=7.5$ $\mathrm{Hz}, 1.0,2 \mathrm{H}, \mathrm{CH}$ Fmoc), 5.91 (ddt, $J=17.0,10.2,5.7 \mathrm{~Hz}, 1 \mathrm{H}, \mathrm{CH}=\mathrm{CH}_{2}$ ), 5.16 (dd, $J=17.2,1.6$ $\left.\mathrm{Hz}, 1 \mathrm{H}, \mathrm{CH}=\mathrm{CH}_{2 \mathrm{a}}\right), 5.25\left(\mathrm{dd}, J=10.4,1.2 \mathrm{~Hz}, 1 \mathrm{H}, \mathrm{CH}=\mathrm{CH}_{2 \mathrm{~b}}\right), 5.22(\mathrm{t}, J=7.6 \mathrm{~Hz}, 1 \mathrm{H}, \mathrm{C}=\mathrm{C} \underline{\mathrm{H}}-$ $\mathrm{CH}_{2}-\mathrm{S} \mathrm{GG}$ ), 5.07-5.12 (m, 3H, 3 * $\mathrm{CH}$ GG), 4.66 (dd, $J=5.6 \mathrm{~Hz}, 2 \mathrm{H}, \mathrm{OCH}_{2} \mathrm{OAll}$ ), 4.60-4.62 (m, 2H, $\alpha-C H$ Cys, $\alpha-C H$ Ser), $4.40\left(\mathrm{~d}, J=6.8 \mathrm{~Hz}, 2 \mathrm{H}, \mathrm{OCH}_{2} \mathrm{Fmoc}\right.$ ), 4.26 (dd, $J=5.2 \mathrm{~Hz}$, $1 \mathrm{H}, \alpha-\mathrm{CH}$ Ser'), 4.23 (t, $J=6.8 \mathrm{~Hz}, 1 \mathrm{H}, \mathrm{CHCH}_{2} \mathrm{O} \mathrm{Fmoc}$ ), 3.94 (dd, $J=11.6,4.0 \mathrm{~Hz}, 1 \mathrm{H}, \beta-\mathrm{CH}_{2 \mathrm{a}}$ Ser), 3.89-3.93 (m, $1 \mathrm{H}, \beta-\mathrm{CH}_{2 \mathrm{a}}$ Ser'), 3.85 (dd, $J=11.8,3.4 \mathrm{~Hz}, 1 \mathrm{H}, \beta-\mathrm{CH}_{2 \mathrm{~b}}$ Ser), 3.69 (dd, $J=$ 11.0, 7.0 Hz, $1 \mathrm{H}, \beta-\mathrm{CH}_{2 \mathrm{~b}}$ Ser'), 3.22 (dd, $J=12.8,7.6 \mathrm{~Hz}, 1 \mathrm{H}, \mathrm{CH}_{2 \mathrm{a}}-\mathrm{S} \mathrm{GG}$ ), 3.17 (dd, $J=13.0$, $7.8 \mathrm{~Hz}, 1 \mathrm{H}, \mathrm{CH}_{2 b}-\mathrm{S} \mathrm{GG}$ ), 2.99 (dd, $J=14.0,5.6 \mathrm{~Hz}, 1 \mathrm{H}, \beta-\mathrm{CH}_{2 a}$ Cys), 2.85 (dd, $J=13.8,7.8$ $\mathrm{Hz}, 1 \mathrm{H}, \beta-\mathrm{CH}_{2 b}$ Cys), 1.95-2.11 (m, $\left.12 \mathrm{H}, 6{ }^{*} \mathrm{CH}_{2} \mathrm{GG}\right), 1.68\left(\mathrm{~s}, 3 \mathrm{H}, \mathrm{CH}_{3}\right), 1.66\left(\mathrm{~s}, 3 \mathrm{H}, \mathrm{CH}_{3}\right)$, $1.59\left(\mathrm{~s}, 9 \mathrm{H}, 3{ }^{*} \mathrm{CH}_{3}\right) .{ }^{13} \mathrm{C} \mathrm{NMR}\left(100.7 \mathrm{MHz}, \mathrm{CDCl}_{3} / \mathrm{CD}_{3} \mathrm{OD} 10: 1\right): \delta 170.88,170.51,169.27$, 156.42 , 143.30, 143.18, 140.77, 139.38, 134.67, 134.27, 131.05, 130.52, 127.19, 126.54, $124.49,123.83,123.70,123.31,119.36,119.07,117.80,66.71,65.59,61.81,61.32,56.04$, $54.54,52.44,46.56,39.15,39.11,39.07,32.03,29.17,26.17,26.03,25.91,24.82,16.80$, 15.27, 15.17, 15.15. HRMS (FAB; 3-NBA) $\mathrm{m} / \mathrm{z}$ : calcd for $(\mathrm{M}+\mathrm{H})^{+} \mathrm{C}_{47} \mathrm{H}_{64} \mathrm{~N}_{3} \mathrm{O}_{8} \mathrm{~S} 830.4414$, found 830.4399 . 


\section{N-9-Fluorenylmethyloxycarbonyl-L-seryl-S-geranylgeranyl-L-cysteyl-L-serine}

(Fmoc-Ser-Cys(GG)-Ser-OH) (27). This compound was prepared by means of the standard allyl ester removal procedure with the tripeptide Fmoc-Ser-Cys(GG)-Ser-All 26 (110 mg, 0.13 $\mathrm{mmol})$. The compound was purified by flash chromatography on silica gel using a gradient elution starting with dichloromethane/methanol 30:1, then 10:1, and finally 5:1. After purification $99 \mathrm{mg}(95 \%)$ of the desired product 27 were obtained as a white solid. $R_{f}=0.05$ (dichloromethane/methanol 10:1). $\mathrm{mp}=156-158^{\circ} \mathrm{C}$. $[\alpha]^{20} \mathrm{D}=-15.9$ (c=1.0, $\mathrm{CHCl}_{3} / \mathrm{CH}_{3} \mathrm{OH}$ 1:1). ${ }^{1} \mathrm{H}$ NMR (400 MHz, $\left.\mathrm{CDCl}_{3} / \mathrm{CD}_{3} \mathrm{OD} 10: 1\right): \delta 7.76(\mathrm{~d}, J=7.6 \mathrm{~Hz}, 2 \mathrm{H}, \mathrm{CH}$ Fmoc), 7.60-7.63 (m, 2H, CH Fmoc), 7.40 (dd, J=7.4 Hz, 2H, CH Fmoc), 7.31 (dd, J=7.4 Hz, 2H, CH Fmoc), $5.22\left(\mathrm{t}, \mathrm{J}=7.0 \mathrm{~Hz}, 1 \mathrm{H}, \mathrm{C}=\mathrm{C} \underline{\mathrm{H}}-\mathrm{CH}_{2}-\mathrm{S} \mathrm{GG}\right), 5.07-5.13\left(\mathrm{~m}, 3 \mathrm{H}, 3{ }^{*} \mathrm{CH} \mathrm{GG}\right), 4.64(\mathrm{dd}, \mathrm{J}=6.0 \mathrm{~Hz}$, $1 \mathrm{H}, \alpha-\mathrm{CH}$ Cys), 4.53 (br, $1 \mathrm{H}, \alpha-\mathrm{CH}$ Ser), 4.39 (d, J=6.8 Hz, 2H, OCH ), 4.31 (dd, $J=4.8 \mathrm{~Hz}$, $1 \mathrm{H}, \alpha-\mathrm{CH}$ Ser'), 4.22 (t, J=6.8 Hz, 1H, $\underline{\mathrm{HCH}}_{2} \mathrm{O} \mathrm{Fmoc}$ ), 3.95 (dd, J=11.4, $3.4 \mathrm{~Hz}, 1 \mathrm{H}, \beta-\mathrm{CH}_{2 \mathrm{a}}$ Ser), 3.83-3.92 (m, $2 \mathrm{H}, \beta-\mathrm{CH}_{2 \mathrm{a}}$ Ser', $\beta-\mathrm{CH}_{2 \mathrm{~b}}$ Ser), 3.73 (dd, J = 10.8, $7.2 \mathrm{~Hz}, 1 \mathrm{H}, \beta-\mathrm{CH}_{2 b}$ Ser') $^{\prime}$, 3.15-3.25 (m, 2H, CH $\mathrm{CH}_{2} \mathrm{SG}$ ), 3.01 (dd, $J=13.6,5.2 \mathrm{~Hz}, 1 \mathrm{H}, \beta-\mathrm{CH}_{2 \mathrm{a}}$ Cys), 2.85 (dd, $J=13.8$, $8.2 \mathrm{~Hz}, 1 \mathrm{H}, \beta-\mathrm{CH}_{2 \mathrm{~b}}$ Cys), 1.94-2.10 (m, 12H, $\left.6{ }^{*} \mathrm{CH}_{2} \mathrm{GG}\right), 1.68\left(\mathrm{~s}, 3 \mathrm{H}, \mathrm{CH}_{3}\right), 1.66$ (s, 3H, $\left.\mathrm{CH}_{3}\right), 1.60\left(\mathrm{~s}, 9 \mathrm{H}, 3{ }^{*} \mathrm{CH}_{3}\right) .{ }^{13} \mathrm{C} \operatorname{NMR}\left(100.7 \mathrm{MHz}, \mathrm{CDCl}_{3} / \mathrm{CD}_{3} \mathrm{OD} 10: 1\right): \delta$ 175.29, 170.97, 170.39 , 156.37, 143.31, 143.19, 140.76, 139.33, 134.64, 134.24, 130.49, 127.16, 126.52, 124.48, 123.80, 123.68, 123.31, 119.33, 119.06, 66.67, 61.82, 61.45, 55.98, 54.55, 52.56, 46.55, 39.13, 39.10, 39.05, 32.01, 29.14, 26.16, 26.01, 25.88, 24.77, 16.74, 15.21, 15.11, 15.09. HRMS (FAB; triethanolamine) $\mathrm{m} / \mathrm{z}$ : calcd for $(\mathrm{M}-\mathrm{H})^{-} \mathrm{C}_{44} \mathrm{H}_{58} \mathrm{~N}_{3} \mathrm{O}_{8} \mathrm{~S} 788.3945$, found 788.3967.

\section{N-9-Fluorenylmethyloxycarbonyl-L-seryl-S-geranylgeranyl-L-cysteyl-L-seryl-S- geranylgeranyl-L-cysteine methyl ester (Fmoc-Ser-Cys(GG)-Ser-Cys(GG)-OMe) (28). This} compound was prepared by means of the standard peptide coupling procedure with the tripeptide Fmoc-Ser-Cys(GG)-Ser-OH 27 (99 mg, 0.13 mmol, 1 eq.) and H-Cys(GG)-OMe 10a (62 mg, $0.15 \mathrm{mmol})$. Purification on silica gel by flash chromatography with dichloromethane/methanol 100:1, then 40:1 as eluent yielded $112 \mathrm{mg}(75 \%)$ of the desired product 28 as a white solid. $R_{f}=0.48$ (dichloromethane/methanol $10: 1$ ). $\mathrm{mp}=170-171^{\circ} \mathrm{C} \cdot \mathrm{t}_{\mathrm{R}}=$ $26.17 \min .[\alpha]^{20}{ }_{D}=-28.4\left(c=1.0, \mathrm{CHCl}_{3} / \mathrm{CH}_{3} \mathrm{OH} 1: 1\right) .{ }^{1} \mathrm{H} \mathrm{NMR}\left(400 \mathrm{MHz}, \mathrm{CDCl}_{3} / \mathrm{CD}_{3} \mathrm{OD}\right.$ 10:1): $\delta 7.77$ (d, J=7.6 Hz, 2H, CH Fmoc), 7.61 (d, J=7.2 Hz, 2H, CH Fmoc), 7.40 (dd, $J=$ $7.6 \mathrm{~Hz}, 2 \mathrm{H}, \mathrm{CH}$ Fmoc), 7.32 (dd, J=7.6 Hz, 2H, CH Fmoc), 5.22 (t, J= $7.6 \mathrm{~Hz}, 1 \mathrm{H}, \mathrm{C}=\mathrm{CH}-$ $\mathrm{CH}_{2}-\mathrm{S}$ GG), 5.20 (t, J=7.8 Hz, 1H, CH GG'), 5.07-5.12 (m, $\left.6 \mathrm{H}, 6{ }^{*} \mathrm{CH} \mathrm{GG}\right), 4.67$ (dd, $J=7.8$, $5.0 \mathrm{~Hz}, 1 \mathrm{H}, \alpha-\mathrm{CH}$ Cys), 4.59 (dd, J = $6.4 \mathrm{~Hz}, 1 \mathrm{H}, \alpha-\mathrm{CH}$ Cys'), 4.51 (dd, J = $4.6 \mathrm{~Hz}, 1 \mathrm{H}, \alpha-\mathrm{CH}$ Ser), $4.40\left(\mathrm{~d}, J=6.8 \mathrm{~Hz}, 2 \mathrm{H}, \mathrm{OCH}_{2}\right), 4.32(\mathrm{dd}, J=5.8 \mathrm{~Hz}, 1 \mathrm{H}, \alpha-\mathrm{CH}$ Ser'), 4.22 (t, J = $6.8 \mathrm{~Hz}$, $\left.1 \mathrm{H}, \mathrm{CHCH}_{2} \mathrm{O} \mathrm{Fmoc}\right)$, 3.89-3.95 (m, 2H, 2 * $\left.\beta-\mathrm{CH}_{2 \mathrm{a}} \mathrm{Ser}\right), 3.76\left(\mathrm{~s}, 3 \mathrm{H}, \mathrm{OCH}_{3}\right), 3.67-3.76(\mathrm{~m}, 2 \mathrm{H}$, 2 * $\beta-\mathrm{CH}_{2 b}$ Ser), 3.23 (dd, $J=12.8,8.0 \mathrm{~Hz}, 1 \mathrm{H}, \mathrm{CH}_{2 \mathrm{a}}-\mathrm{S} \mathrm{GG}$ ), 3.20 (d, J = $7.6 \mathrm{~Hz}, 2 \mathrm{H}, \mathrm{CH}_{2}-\mathrm{S}$ GG'), 3.12 (dd, $J=13.0,7.4 \mathrm{~Hz}, 1 \mathrm{H}, \mathrm{CH}_{2 \mathrm{~b}}-\mathrm{S} \mathrm{GG}$ ), 2.95-3.02 (m, $2 \mathrm{H}, 2$ * $\beta-\mathrm{CH}_{2 \mathrm{a}}$ Cys), 2.88 (dd, $J=14.0,7.6 \mathrm{~Hz}, 1 \mathrm{H}, \beta-\mathrm{CH}_{2 b}$ Cys), 2.82 (dd, $J=13.8,7.8 \mathrm{~Hz}, 1 \mathrm{H}, \beta-\mathrm{CH}_{2 b}$ Cys'), 1.95-2.11 (m, $\left.24 \mathrm{H}, 12{ }^{*} \mathrm{CH}_{2} \mathrm{GG}\right), 1.67\left(\mathrm{~s}, 12 \mathrm{H}, 4{ }^{*} \mathrm{CH}_{3}\right), 1.60\left(\mathrm{~s}, 18 \mathrm{H}, 6{ }^{*} \mathrm{CH}_{3}\right) .{ }^{13} \mathrm{C} \mathrm{NMR}(100.7 \mathrm{MHz}$, $\left.\mathrm{CDCl}_{3} / \mathrm{CD}_{3} \mathrm{OD} 10: 1\right): \delta 171.32,170.99,170.63,170.23,156.32,143.61,143.48,141.12$, $140.16,140.09,135.26,135.23,134.80,134.79,131.09,127.60,126.94,124.89,124.21$, 124.04, 124.02, 123.59, 123.58, 119.82, 119.19, 119.11, 67.14, 62.52, 62.00, 55.70, 54.96, 53.24, 52.46, 52.03, 46.91, 39.55, 39.53, 39.49, 32.46, 32.31, 29.64, 29.42, 26.59, 26.49, 26.48, 26.37, 26.35, 25.46, 17.45, 15.93, 15.91, 15.80, 15.78. FAB-MS m/z: calcd for $(\mathrm{M}+\mathrm{H})^{+}$ $\mathrm{C}_{68} \mathrm{H}_{99} \mathrm{~N}_{4} \mathrm{O}_{9} \mathrm{~S}_{2}$ 1179.7, found 1179.6; calcd for $(\mathrm{M}+\mathrm{Na})^{+} \mathrm{C}_{68} \mathrm{H}_{98} \mathrm{~N}_{4} \mathrm{NaO}_{9} \mathrm{~S}_{2}$ 1201.7, found 1201.6 . 
L-Seryl-S-geranylgeranyl-L-cysteyl-L-seryl-S-geranylgeranyl-L-cysteine methyl ester (H-Ser-Cys(GG)-Ser-Cys(GG)-OMe) (19c). At room temperature, a solution of Fmoc-SerCys(GG)-Ser-Cys(GG)-OMe 28 (50 mg, $42 \mu \mathrm{mol})$ in dry dichloromethane/diethylamine 4:1 (1.0 $\mathrm{mL}$ ) under an argon atmosphere was stirred for $2.5 \mathrm{~h}$. The solvent was removed by coevaporation with toluene $(2 * 30 \mathrm{~mL})$ and chloroform $(30 \mathrm{~mL})$. The compound was purified by flash chromatography on silica gel using a gradient elution starting with cyclohexane/ethyl acetate $1: 1$, followed by dichloromethane/methanol $50: 1$, then $25: 1$ and finally $10: 1$. After purification, $37 \mathrm{mg}(92 \%)$ of the desired product $19 \mathrm{c}$ were obtained as a white solid. $R_{f}=0.18$ (dichloromethane/methanol 10:1). $\mathrm{mp}=96-97^{\circ} \mathrm{C} .[\alpha]^{20}{ }_{\mathrm{D}}=-27.5\left(c=0.75, \mathrm{CHCl}_{3} / \mathrm{CH}_{3} \mathrm{OH} 1: 1\right)$. ${ }^{1} \mathrm{H}$ NMR $\left(400 \mathrm{MHz}, \mathrm{CDCl}_{3} / \mathrm{CD}_{3} \mathrm{OD} 10: 1\right): \delta 5.24$ (t, $\left.J=7.4 \mathrm{~Hz}, 1 \mathrm{H}, \mathrm{C}=\mathrm{CH}-\mathrm{CH}_{2}-\mathrm{S} \mathrm{GG}\right), 5.20$ (t, $J=7.8 \mathrm{~Hz}, 1 \mathrm{H}, \mathrm{C}=\mathrm{C} \underline{\mathrm{H}}-\mathrm{CH}_{2}-\mathrm{S} \mathrm{GG}$ ), 5.07-5.13 (m, $\left.6 \mathrm{H}, 6{ }^{*} \mathrm{CH} \mathrm{GG}\right), 4.68$ (dd, $J=8.0,4.8 \mathrm{~Hz}$, $1 \mathrm{H}, \alpha-\mathrm{CH}$ Cys), 4.55 (dd, $J=6.2,7.0 \mathrm{~Hz}, 1 \mathrm{H}, \alpha-\mathrm{CH}$ Cys'), 4.51 (dd, $J=4.6 \mathrm{~Hz}, 1 \mathrm{H}, \alpha-\mathrm{CH}$ Ser), 3.98 (dd, $J=11.6,4.4 \mathrm{~Hz}, 1 \mathrm{H}, \beta-\mathrm{CH}_{2 \mathrm{a}}$ Ser), 3.81 (dd, $J=11.6,4.4 \mathrm{~Hz}, 1 \mathrm{H}, \beta-\mathrm{CH}_{2 \mathrm{a}}$ Ser'), 3.75 (s, $3 \mathrm{H}, \mathrm{OCH}_{3}$ ), 3.71 (dd, $J=11.8,5.0 \mathrm{~Hz}, 1 \mathrm{H}, \beta-\mathrm{CH}_{2 b} \mathrm{Ser}$ ), 3.66 (dd, $J=10.8,6.4 \mathrm{~Hz}, 1 \mathrm{H}, \beta-$ $\mathrm{CH}_{2 b}$ Ser'), 3.51 (dd, $J=5.4 \mathrm{~Hz}, 1 \mathrm{H}, \alpha-\mathrm{CH}$ Ser'), 3.21-3.26 (m, 1H, $\left.\mathrm{CH}_{2 \mathrm{a}}-\mathrm{S} \mathrm{GG}\right), 3.22$ (d, $J=$ $8.4 \mathrm{~Hz}, 2 \mathrm{H}, \mathrm{CH}_{2}-\mathrm{S}$ GG'), 3.12 (dd, $J=13.2,7.2 \mathrm{~Hz}, 1 \mathrm{H}, \mathrm{CH}_{2 b}-\mathrm{S} \mathrm{GG}$ ), 2.98 (dd, $J=13.8,5.8$ $\mathrm{Hz}, 1 \mathrm{H}, \beta-\mathrm{CH}_{2 \mathrm{a}}$ Cys), 2.97 (dd, $J=13.8,4.6 \mathrm{~Hz}, 1 \mathrm{H}, \beta-\mathrm{CH}_{2 \mathrm{a}}$ Cys'), 2.89 (dd, $J=13.6,7.6 \mathrm{~Hz}$, $1 \mathrm{H}, \beta-\mathrm{CH}_{2 b}$ Cys), 2.82 (dd, $J=14.0,8.0 \mathrm{~Hz}, 1 \mathrm{H}, \beta-\mathrm{CH}_{2 b}$ Cys'), 1.95-2.12 (m, 24H, 12 * $\mathrm{CH}_{2}$ $\mathrm{GG}), 1.68\left(\mathrm{~s}, 12 \mathrm{H}, 4{ }^{*} \mathrm{CH}_{3}\right), 1.60\left(\mathrm{~s}, 18 \mathrm{H}, 6{ }^{*} \mathrm{CH}_{3}\right) .{ }^{13} \mathrm{C} \mathrm{NMR}\left(100.7 \mathrm{MHz}, \mathrm{CDCl}_{3} / \mathrm{CD}_{3} \mathrm{OD}\right.$ $10: 1): \delta 174.79,171.04,170.68,170.32,140.26,140.22,135.36,134.90,131.19,124.29$, 124.10, 124.09, 123.64, 119.21, 64.66, 62.13, 55.90, 54.83, 53.04, 52.59, 51.99, 39.62, 39.59, 39.57, 32.72, 32.60, 29.74, 29.51, 26.67, 26.58, 26.56, 26.43, 25.56, 17.56, 16.06, 16.02, 15.91, 15.89. HRMS (FAB; 3-NBA) $\mathrm{m} / z$ : calcd for $(\mathrm{M}+\mathrm{Na})^{+} \mathrm{C}_{53} \mathrm{H}_{88} \mathrm{~N}_{4} \mathrm{NaO}_{7} \mathrm{~S}_{2} 979.5992$, found 979.5970 .

\section{$N$-9-Fluorenylmethyloxycarbonyl-S-tert-butylthio-L-cysteyl- $N^{(\varepsilon-5-d i m e t h y l a m i n o-~}$} naphthalene-1-sulfonyl-L-lysyl-L-seryl-S-tert-butylthio-L-cysteyl-L-seryl-Sgeranylgeranyl-L-cysteine methyl ester (Fmoc-Cys( $\left.{ }^{t} B u\right)-L y s(d a n s)-S e r-C y s\left(S^{t} B u\right)-S e r-$ Cys(GG)-OMe) (29a). This compound was prepared by means of the standard peptide coupling procedure with the dipeptide Fmoc-Cys( $\left.\mathrm{S}^{t} \mathrm{Bu}\right)-\mathrm{Lys}($ dansyl)- $\mathrm{OH} 7(42 \mathrm{mg}, 52 \mu \mathrm{mol})$ and the tetrapeptide $\mathrm{H}$-Ser-Cys $\left(\mathrm{S}^{t} \mathrm{Bu}\right)$-Ser-Cys(GG)-OMe 19a (38 mg, $49 \mu \mathrm{mol}$, 1 eq.). Purification on silica gel by flash chromatography with dichloromethane/methanol 30:1 as eluent yielded $56 \mathrm{mg}(74 \%)$ of the desired product 29 a as a pale yellowish-green solid. $R_{f}=$ 0.63 (dichloromethane/methanol 10:1). $\mathrm{mp}=206^{\circ} \mathrm{C} . \mathrm{t}_{\mathrm{R}}=24.79 \mathrm{~min} .[\alpha]^{20}{ }_{\mathrm{D}}=+56.0(c=1.0$, $\mathrm{CHCl}_{3}$ ). ${ }^{1} \mathrm{H} \mathrm{NMR}\left(400 \mathrm{MHz}, \mathrm{CDCl}_{3} / \mathrm{CD}_{3} \mathrm{OD} 10: 1\right): \delta 8.51$ (d, $J=8.4 \mathrm{~Hz}, 1 \mathrm{H}, \mathrm{CH}-2$ dansyl), 8.31 (d, $J=8.8 \mathrm{~Hz}, 1 \mathrm{H}, \mathrm{CH}-8$ dansyl), 8.17 (dd, $J=7.4,1.4 \mathrm{~Hz}, 1 \mathrm{H}, \mathrm{CH}-4$ dansyl), $7.76(\mathrm{~d}, J=8.0$ $\mathrm{Hz}, 2 \mathrm{H}, \mathrm{CH}$ Fmoc), 7.59-7.63 (m, 2H, CH Fmoc), 7.56 (dd, $J=8.6,7.8 \mathrm{~Hz}, 1 \mathrm{H}, \mathrm{CH}-7$ dansyl), 7.50 (dd, $J=8.6,7.4 \mathrm{~Hz}, 1 \mathrm{H}, \mathrm{CH}-3$ dansyl), 7.36-7.41 (m, 2H, CH Fmoc), 7.27-7.31 (m, 2H, CH Fmoc), 7.19 (d, $J=7.2 \mathrm{~Hz}, 1 \mathrm{H}, \mathrm{CH}-6$ dansyl), 5.18-5.22 (m, $\left.1 \mathrm{H}, \mathrm{C}=\mathrm{C} \underline{\mathrm{H}}-\mathrm{CH}_{2}-\mathrm{S} \mathrm{GG}\right), 5.08-$ $5.12(\mathrm{~m}, 3 \mathrm{H}, 3$ * $\mathrm{CH} \mathrm{GG}), 4.73\left(\mathrm{dd}, J=8.8,4.8 \mathrm{~Hz}, 1 \mathrm{H}, \alpha-\mathrm{CH}\right.$ Cys $\left.\mathrm{SABu}_{\mathrm{B}}\right), 4.67$ (dd, $J=7.8,5.4$ $\mathrm{Hz}, 1 \mathrm{H}, \alpha-\mathrm{CH}$ Cys(GG)), 4.38 (dd, $J=5.4,1 \mathrm{H}, \alpha-\mathrm{CH}$ Ser), 4.32-4.52 (m, 4H, $\alpha-\mathrm{CH}$ Ser, $\alpha-\mathrm{CH}$ Cys ${ }_{\mathrm{StBu}}, \mathrm{OCH}_{2} \mathrm{Fmoc}$ ), 4.20-4.24 (m, 2H, $\alpha-\mathrm{CH}$ Lys, $\mathrm{CHCH}_{2} \mathrm{O}$ Fmoc), 3.91 (dd, $J=11.6,5.6$ $\mathrm{Hz}, 2 \mathrm{H}, 2$ * $\beta-\mathrm{CH}_{2 \mathrm{a}}$ Ser), 3.81 (dd, $J=11.4,5.2 \mathrm{~Hz}, 2 \mathrm{H}, 2$ * $\left.\beta-\mathrm{CH}_{2 \mathrm{~b}} \mathrm{Ser}\right), 3.74\left(\mathrm{~s}, 3 \mathrm{H}, \mathrm{OCH}_{3}\right)$, 3.00-3.28 (m, 6H, CH $\mathrm{CH}_{2} \mathrm{~S} \mathrm{GG}, 2$ * $\left.\beta-\mathrm{CH}_{2} \mathrm{Cys}_{\mathrm{StBu}}\right), 2.95$ (dd, $J=14.0,5.2 \mathrm{~Hz}, 1 \mathrm{H}, \beta-\mathrm{CH}_{2 \mathrm{a}}$ Cys(GG)), 2.87 (s, 6H, N( $\left.\left.\mathrm{CH}_{3}\right)_{2}\right), 2.84-2.93\left(\mathrm{~m}, 2 \mathrm{H}, \varepsilon-\mathrm{CH}_{2} \mathrm{Lys}\right), 2.81$ (dd, $J=13.8,7.8 \mathrm{~Hz}, 1 \mathrm{H}$, $\beta-\mathrm{CH}_{2 b}$ Cys(GG)), $1.95-2.12\left(\mathrm{~m}, 12 \mathrm{H}, 6{ }^{*} \mathrm{CH}_{2} \mathrm{GG}\right), 1.71-1.83\left(\mathrm{~m}, 1 \mathrm{H}, \beta-\mathrm{CH}_{2 \mathrm{a}} \mathrm{Lys}\right), 1.68$ (d, $\left.J=1.2 \mathrm{~Hz}, 3 \mathrm{H}, \mathrm{CH}_{3}\right), 1.67\left(\mathrm{~d}, J=1.2 \mathrm{~Hz}, 3 \mathrm{H}, \mathrm{CH}_{3}\right), 1.60-1.68\left(\mathrm{~m}, 1 \mathrm{H}, \beta-\mathrm{CH}_{2 \mathrm{~b}} \mathrm{Lys}\right), 1.60$ (s, $9 \mathrm{H}, 3$ * $\left.\mathrm{CH}_{3}\right), 1.39-1.48\left(\mathrm{~m}, 4 \mathrm{H}, \delta-, \gamma-\mathrm{CH}_{2} \mathrm{Lys}\right), 1.35\left(\mathrm{~s}, 9 \mathrm{H}, \mathrm{C}\left(\mathrm{CH}_{3}\right)_{3}\right), 1.32\left(\mathrm{~s}, 9 \mathrm{H}, \mathrm{C}\left(\mathrm{CH}_{3}\right)_{3}\right)$. ${ }^{13} \mathrm{C}$ NMR $\left(100.7 \mathrm{MHz}, \mathrm{CDCl}_{3} / \mathrm{CD}_{3} \mathrm{OD} 10: 1\right): \delta 172.60,171.70,170.99,170.82,170.49,170.03$, 
$156.63,151.61,143.47,143.38,141.02,139.85,135.14,134.89,134.72,131.02,129.99$, $129.66,129.39,128.87,127.99,127.52,126.92,126.87,124.92,124.89,124.14,123.97$, $123.57,122.95,119.71,119.24,118.73,115.02,67.23,61.94,61.40,55.95,55.32,54.65$, $54.15,53.13,52.31,51.96,48.21,48.03,46.78,45.12,41.96,41.38,40.71,39.47,32.40$, $30.27,29.55,29.33,28.53,26.53,26.42,26.32,25.36,21.95,17.36,15.81,15.71,15.69$. ESIMS $\mathrm{m} / \mathrm{z}$ : calcd for $(\mathrm{M}+\mathrm{H})^{+} \mathrm{C}_{77} \mathrm{H}_{111} \mathrm{~N}_{8} \mathrm{O}_{13} \mathrm{~S}_{6}$ 1547.7, found 1547.6; calcd for $(\mathrm{M}+\mathrm{Na})^{+}$ $\mathrm{C}_{77} \mathrm{H}_{110} \mathrm{~N}_{8} \mathrm{NaO}_{13} \mathrm{~S}_{6}$ 1569.6, found 1569.7; calcd for $(\mathrm{M}+\mathrm{K})^{+} \mathrm{C}_{77} \mathrm{H}_{110} \mathrm{~N}_{8} \mathrm{KO}_{13} \mathrm{~S}_{6}$ 1585.6, found 1585.6 .

\section{$N$-9-Fluorenylmethyloxycarbonyl-S-tert-butylthio-L-cysteyl- $N^{(\varepsilon-5-d i m e t h y l a m i n o-~}$ naphthalene-1-sulfonyl-L-lysyl-L-seryl-S-geranylgeranyl-L-cysteyl-L-seryl-S-tert-} butylthio-L-cysteine methyl ester (Fmoc-Cys(St Bu)-Lys(dans)-Ser-Cys(GG)-SerCys $\left.\left(S^{t} \mathrm{Bu}\right)-\mathrm{OMe}\right)(29 \mathrm{~b})$. This compound was prepared by means of the standard peptide coupling procedure with the dipeptide Fmoc-Cys(StBu)-Lys(dansyl)-OH 7 (15 mg, $19 \mu \mathrm{mol})$ and the tetrapeptide H-Ser-Cys(GG)-Ser-Cys( $\left.{ }^{t} \mathrm{Bu}\right)-\mathrm{OMe} 19 \mathrm{~b}(14 \mathrm{mg}, 19 \mu \mathrm{mol})$. Purification on silica gel by flash chromatography with dichloromethane/methanol 40:1 as eluent yielded $16 \mathrm{mg}(55 \%)$ of the desired product $29 \mathrm{~b}$ as a pale yellowish-green solid. $R_{f}=0.55$ (dichloromethane/methanol 10:1). $\mathrm{mp}=187-190^{\circ} \mathrm{C} . \mathrm{t}_{\mathrm{R}}=23.87 \mathrm{~min} .[\alpha]^{20}{ }_{\mathrm{D}}=+23.6(c=0.5$, $\mathrm{CHCl}_{3}$ ). ${ }^{1} \mathrm{H}$ NMR $\left(500 \mathrm{MHz}, \mathrm{CDCl}_{3} / \mathrm{CD}_{3} \mathrm{OD} 10: 1\right): \delta 8.52(\mathrm{~d}, J=8.5 \mathrm{~Hz}, 1 \mathrm{H}, \mathrm{CH}-2$ dansyl), 8.30 (d, $J=8.5 \mathrm{~Hz}, 1 \mathrm{H}, \mathrm{CH}-8$ dansyl), 8.18 (dd, $J=7.5,1.0 \mathrm{~Hz}, 1 \mathrm{H}, \mathrm{CH}-4$ dansyl), $7.76(\mathrm{~d}, J=7.5$ $\mathrm{Hz}, 2 \mathrm{H}, \mathrm{CH}$ Fmoc), 7.60-7.64 (m, 2H, CH Fmoc), 7.56 (dd, $J=8.5,7.5 \mathrm{~Hz}, 1 \mathrm{H}, \mathrm{CH}-7$ dansyl), 7.50 (dd, $J=8.5,7.5 \mathrm{~Hz}, 1 \mathrm{H}, \mathrm{CH}-3$ dansyl), 7.37-7.41 (m, 2H, CH Fmoc), 7.27-7.31 (m, 2H, CH Fmoc), 7.20 (d, $J=7.5 \mathrm{~Hz}, 1 \mathrm{H}, \mathrm{CH}-6$ dansyl), 5.23 (t, $J=7.5 \mathrm{~Hz}, 1 \mathrm{H}, \mathrm{C}=\mathrm{CH}_{-}-\mathrm{CH}_{2}-\mathrm{S} \mathrm{GG}$ ), 5.08-5.13 (m, 3H, 3 * $\mathrm{CH} \mathrm{GG}$ ), 4.78 (dd, $J=7.3,5.3 \mathrm{~Hz}, 1 \mathrm{H}, \alpha-\mathrm{CH}$ Cys ${ }_{\mathrm{StB}}$ ), 4.59 (dd, $J=8.0$, $5.5 \mathrm{~Hz}, 1 \mathrm{H}, \alpha-\mathrm{CH}$ Cys(GG)), 4.51 (dd, $J=5.3,4.8 \mathrm{~Hz}, 1 \mathrm{H}, \alpha-\mathrm{CH}$ Ser), 4.41 (dd, $J=5.3 \mathrm{~Hz}$, $1 \mathrm{H}, \alpha-\mathrm{CH}$ Ser'), 4.34-4.49 (m, 3H, $\alpha-\mathrm{CH}$ Cys $_{\mathrm{StB}}$ ', $\left.\mathrm{OCH}_{2} \mathrm{Fmoc}\right), 4.20-4.25$ (m, 2H, $\alpha-\mathrm{CH}$ Lys, $\mathrm{CHCH}_{2} \mathrm{O}$ Fmoc), 3.89-3.94 (m, 2H, 2 * $\left.\beta-\mathrm{CH}_{2 a} \mathrm{Ser}\right), 3.79-3.83$ (m, 2H, $\left.2{ }^{*} \beta-\mathrm{CH}_{2 b} \mathrm{Ser}\right), 3.75$ (s, $3 \mathrm{H}, \mathrm{OCH}_{3}$ ), 3.15-3.25 (m, 4H, $\mathrm{CH}_{2}-\mathrm{S}$ GG, $2{ }^{*} \beta-\mathrm{CH}_{2 \mathrm{a}} \mathrm{CyS}_{\mathrm{StBu}}$ ), 3.09 (dd, $J=14.0,7.5 \mathrm{~Hz}, 1 \mathrm{H}$, $\beta-\mathrm{CH}_{2 b}$ Cys $\left._{\mathrm{stBu}}\right), 2.98-3.05$ (m, 2H, $\left.\beta-\mathrm{CH}_{2 \mathrm{a}} \mathrm{Cys}(\mathrm{GG}), \beta-\mathrm{CH}_{2 b} \mathrm{Cys}_{\mathrm{SABu}}{ }^{\prime}\right), 2.88\left(\mathrm{~s}, 6 \mathrm{H}, \mathrm{N}\left(\mathrm{CH}_{3}\right)_{2}\right)$, 2.84-2.92 (m, 3H, $\varepsilon-\mathrm{CH}_{2}$ Lys, $\beta-\mathrm{CH}_{2 b}$ Cys(GG)), $1.95-2.11\left(\mathrm{~m}, 12 \mathrm{H}, 6{ }^{*} \mathrm{CH}_{2} \mathrm{GG}\right), 1.68(\mathrm{~d}, J=$ $\left.1.0 \mathrm{~Hz}, 3 \mathrm{H}, \mathrm{CH}_{3}\right), 1.67\left(\mathrm{~s}, 3 \mathrm{H}, \mathrm{CH}_{3}\right), 1.60\left(\mathrm{~s}, 9 \mathrm{H}, 3{ }^{*} \mathrm{CH}_{3}\right), 1.56-1.80\left(\mathrm{~m}, 2 \mathrm{H}, \beta-\mathrm{CH}_{2} \mathrm{Lys}\right), 1.41-$ $1.48\left(\mathrm{~m}, 4 \mathrm{H}, \delta-, \gamma-\mathrm{CH}_{2}\right.$ Lys), $1.35\left(\mathrm{~s}, 9 \mathrm{H}, \mathrm{C}\left(\mathrm{CH}_{3}\right)_{3}\right), 1.31\left(\mathrm{~s}, 9 \mathrm{H}, \mathrm{C}\left(\mathrm{CH}_{3}\right)_{3}\right) .{ }^{13} \mathrm{C}$ NMR $(100.7$ $\left.\mathrm{MHz}, \mathrm{CDCl}_{3} / \mathrm{CD}_{3} \mathrm{OD} 10: 1\right): \delta 172.55,171.64,170.88,170.80,170.72,170.06,156.60,151.65$, $143.51,143.42,141.06,140.01,135.15,134.87,134.74,131.05,130.03,129.69,129.42$, $128.92,128.02,127.55,126.93,126.91,124.92,124.17,124.01,123.60,122.98,119.74$, $119.11,118.74,115.05,67.26,61.87,61.49,55.90,55.30,54.63,54.15,53.20,52.40,51.93$, $48.24,47.93,46.81,45.15,41.97,41.34,41.22,39.51,39.47,32.22,30.31,29.56,29.47$, $29.48,28.51,26.55,26.47,26.35,25.40,21.91,17.39,15.89,15.74,15.72$. ESI-MS $\mathrm{m} / z$ : calcd for $(\mathrm{M}+\mathrm{H})^{+}, \mathrm{C}_{77} \mathrm{H}_{111} \mathrm{~N}_{8} \mathrm{O}_{13} \mathrm{~S}_{6}$ 1547.7, found 1547.4; calcd for $(\mathrm{M}+\mathrm{Na})^{+} \mathrm{C}_{77} \mathrm{H}_{110} \mathrm{~N}_{8} \mathrm{NaO}_{13} \mathrm{~S}_{6}$ 1569.6, found 1569.5 .

$N$-9-Fluorenylmethyloxycarbonyl-S-tert-butylthio-L-cysteyl- $N^{(\varepsilon-5-d i m e t h y l a m i n o-~}$ naphthalene-1-sulfonyl-L-lysyl-L-seryl-S-geranylgeranyl-L-cysteyl-L-seryl-Sgeranylgeranyl-L-cysteine methyl ester (Fmoc-Cys( $\left.{ }^{t} B u\right)-L y s(d a n s)-S e r-C y s(G G)-S e r-$ Cys(GG)-OMe) (29c). This compound was prepared by means of the standard peptide coupling procedure with the dipeptide Fmoc-Cys( $\left.\mathrm{S}^{t} \mathrm{Bu}\right)-\mathrm{Lys}($ dansyl)-OH 7 (17 mg, $21 \mu \mathrm{mol})$ and the tetrapeptide H-Ser-Cys(GG)-Ser-Cys(GG)-OMe 19c (20 mg, $21 \mu \mathrm{mol})$. Purification on silica gel by flash chromatography with dichloromethane/methanol 100:1, then 40:1 as eluent and further purification by size exclusion chromatography on Sephadex ${ }^{\circledR}$ LH 20 with 
chloroform/methanol $1: 1$ as eluent yielded $20 \mathrm{mg}(56 \%)$ of the desired product $29 \mathrm{c}$ as a pale yellowish-green solid. $R_{f}=0.37$ (dichloromethane/methanol $10: 1$ ). $\mathrm{mp}=196-198^{\circ} \mathrm{C} . \mathrm{t}_{\mathrm{R}}=$ $29.59 \mathrm{~min} .[\alpha]^{20}{ }_{\mathrm{D}}=-6.6\left(c=0.5, \mathrm{CHCl}_{3}\right) .{ }^{1} \mathrm{H} \mathrm{NMR}\left(600 \mathrm{MHz}, \mathrm{CDCl}_{3} / \mathrm{CD}_{3} \mathrm{OD} 10: 1\right): \delta 8.52(\mathrm{~d}, J$ $=9.0 \mathrm{~Hz}, 1 \mathrm{H}, \mathrm{CH}-2$ dansyl), $8.30(\mathrm{~d}, J=9.0 \mathrm{~Hz}, 1 \mathrm{H}, \mathrm{CH}-8$ dansyl), $8.19(\mathrm{~d}, J=7.2 \mathrm{~Hz}, 1 \mathrm{H}, \mathrm{CH}-$ 4 dansyl), 7.74-7.77 (m, 2H, CH Fmoc), 7.58-7.62 (m, 2H, CH Fmoc), 7.56 (dd, J = 8.7 Hz, 1H, $\mathrm{CH}-7$ dansyl), 7.49 (dd, $J=7.8 \mathrm{~Hz}, 1 \mathrm{H}, \mathrm{CH}-3$ dansyl), 7.37-7.41 (m, 2H, CH Fmoc), 7.27-7.31 (m, 2H, CH Fmoc), 7.18 (d, $J=7.8 \mathrm{~Hz}, 1 \mathrm{H}, \mathrm{CH}-6$ dansyl), $5.22\left(\mathrm{t}, J=7.5 \mathrm{~Hz}, 1 \mathrm{H}, \mathrm{C}=\mathrm{CH}_{-}-\mathrm{CH}_{2}-\mathrm{S}\right.$ GG), 5.21 (t, $J=7.5 \mathrm{~Hz}, 1 \mathrm{H}, \mathrm{C}=\mathrm{C} \underline{\mathrm{H}}-\mathrm{CH}_{2}-\mathrm{S} \mathrm{GG}$ ), 5.02-5.13 (m, 6H, 6 * $\left.\mathrm{CH} \mathrm{GG}\right), 4.71$ (dd, $J=$ $6.3 \mathrm{~Hz}, 1 \mathrm{H}, \alpha-\mathrm{CH}$ Cys(GG)), 4.64 (dd, $J=6.6 \mathrm{~Hz}, 1 \mathrm{H}, \alpha-\mathrm{CH}$ Cys(GG)'), 4.50 (dd, $J=4.6 \mathrm{~Hz}$, $1 \mathrm{H}, \alpha-\mathrm{CH}$ Ser), 4.49-4.52 (m, 1H, $\alpha-\mathrm{CH} \mathrm{Cys}_{\mathrm{StBu}}$ ), 4.34-4.47 (m, 3H, $\alpha-\mathrm{CH}$ Ser', $\mathrm{OCH}_{2} \mathrm{Fmoc}$ ), 4.27 (dd, $J=6.3 \mathrm{~Hz}, 1 \mathrm{H}, \alpha-\mathrm{CH}$ Lys), 4.21 (t, $J=6.6 \mathrm{~Hz}, 1 \mathrm{H}, \mathrm{CHCH}_{2} \mathrm{O}$ Fmoc), 3.95-3.99 (m, $2 \mathrm{H}$, 2 * $\beta-\mathrm{CH}_{2 \mathrm{a}}$ Ser), 3.78-3.83 (m, $2 \mathrm{H}, 2$ * $\beta-\mathrm{CH}_{2 b}$ Ser), $3.74\left(\mathrm{~s}, 3 \mathrm{H}, \mathrm{OCH}_{3}\right), 3.17-3.24(\mathrm{~m}, 3 \mathrm{H}$, $\mathrm{CH}_{2 \mathrm{a}}-\mathrm{S}$ GG, $\mathrm{CH}_{2}-\mathrm{S}$ GG'), 3.13-3.16 (m, 1H, $\beta-\mathrm{CH}_{2 \mathrm{a}}$ Cys $\mathrm{StBu}^{\prime}$ ), 3.11 (dd, $J=13.5,6.9 \mathrm{~Hz}, 1 \mathrm{H}$, $\mathrm{CH}_{2 b}-\mathrm{S}$ GG), 3.04-3.07 (m, 2H, $\beta-\mathrm{CH}_{2 b} \mathrm{Cys}_{\mathrm{StBu}}, \beta-\mathrm{CH}_{2 \mathrm{a}} \mathrm{Cys}(\mathrm{GG})$ ), 2.95 (dd, $J=14.4,4.2 \mathrm{~Hz}$, $\left.1 \mathrm{H}, \beta-\mathrm{CH}_{2 \mathrm{a}} \operatorname{Cys}(\mathrm{GG})^{\prime}\right), 2.85-2.90$ (m, 3H, $\varepsilon-\mathrm{CH}_{2}$ Lys, $\beta-\mathrm{CH}_{2 b}$ Cys(GG)), $2.88\left(\mathrm{~s}, 6 \mathrm{H}, \mathrm{N}\left(\mathrm{CH}_{3}\right)_{2}\right)$, $\left.2.82\left(\mathrm{dd}, J=14.1,7.5 \mathrm{~Hz}, 1 \mathrm{H}, \beta-\mathrm{CH}_{2 b} \mathrm{Cys}(\mathrm{GG})\right)^{\prime}\right), 1.96-2.11\left(\mathrm{~m}, 24 \mathrm{H}, 12{ }^{*} \mathrm{CH}_{2} \mathrm{GG}\right), 1.76-1.82$ (m, $1 \mathrm{H}, \beta-\mathrm{CH}_{2 a}$ Lys), 1.69 (s, 6H, 2 * $\left.\mathrm{CH}_{3}\right), 1.67-1.73$ (m, 1H, $\beta-\mathrm{CH}_{2 b}$ Lys), 1.67 (s, 6H, 2 * $\left.\mathrm{CH}_{3}\right), 1.61\left(\mathrm{~s}, 18 \mathrm{H}, 6{ }^{*} \mathrm{CH}_{3}\right), 1.41-1.50\left(\mathrm{~m}, 4 \mathrm{H}, \delta-, \gamma-\mathrm{CH}_{2} \mathrm{Lys}\right), 1.36\left(\mathrm{~s}, 9 \mathrm{H}, \mathrm{C}\left(\mathrm{CH}_{3}\right)_{3}\right) .{ }^{13} \mathrm{C} \mathrm{NMR}$ $\left(100.7 \mathrm{MHz}, \mathrm{CDCl}_{3} / \mathrm{CD}_{3} \mathrm{OD} 10: 1\right): \delta 173.22,172.37,171.53,171.45,170.80,157.37,144.14$, $144.08,141.67,140.38,140.30,135.86,135.61,135.59,135.20,135.19,131.44,130.27$, $130.09,129.48,128.49,128.12,127.51,127.48,125.56,125.51,124.77,124.65,124.64$, $124.29,124.27,123.80,120.29,120.09,119.97,115.93,67.89,62.55,62.20,56.65,56.31$, $55.51,54.90,54.12,52.96,52.90,48.76,47.68,45.86,42.88,42.23,40.32,40.29,33.10$, $33.04,31.26,30.23,30.09,29.54,27.37,27.24,27.21,27.14$,

$27.11,25.97,23.00,17.98,16.56,16.49,16.38,16.34$. ESI-MS $\mathrm{m} / \mathrm{z}$ : calcd for $(\mathrm{M}+\mathrm{H})^{+}$ $\mathrm{C}_{93} \mathrm{H}_{135} \mathrm{~N}_{8} \mathrm{O}_{13} \mathrm{~S}_{5}$ 1731.9, found 1731.7 .

\section{References:}

(1) Perrin, D. D.; Armarego, W. L. F. Purification of Laboratory Chemicals; Pergamon: Oxford, 1988.

(2) Parker, C. W.; Yoo, T. J.; Johnson, M. C.; Godt, S. M. Biochemistry 1967, 6, 3408-. 